WCH-331

Rev. 0

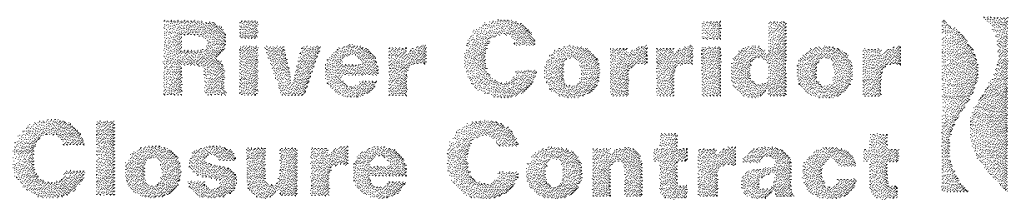

\title{
100-N Area Decision Unit Target Analyte List Development for Soil
}

\section{March 2011}

\section{Washing Ton Closure Hanford}

Prepared for the U.S. Department of Energy, Richland Operations Office Office of Assistant Manager for River Corridor

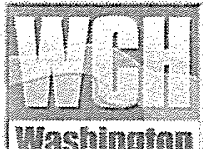




\section{TRADEMARK DISCLAIMER}

Reference herein to any specific commercial product, process, or service by trade name, trademark, manufacturer, or otherwise, does not necessarily constitute or imply its endorsement, recommendation, or favoring by the United States Government or any agency thereof or its contractors or subcontractors.

This report has been reproduced from the best available copy. Available in paper copy.

Available for a processing fee to U.S. Department of Energy and its contractors in paper from:

U.S. Department of Energy

Office of Scientific and Technical Information

P.O. Box 62

Oak Ridge, TN 37831-0062

Telephone: (865) 576-8401

Facsimile: (865) 576-5728

E-mail: reports@osti.gov

Available for sale to the public from:

U.S. Department of Commerce

National Technical Information Service

5285 Port Royal Road

Springfield, VA 22161

Telephone: (800) 553-6847

Facsimile: (703) 605.6900

E-mail: orders@ntis.fedworld.gov

Online ordering: http://www.ntis.gov/ordering.htm

Printed in the United States of America 
DOCUMENT

WCH-331

CONTROL $9 / 18 / 2012 a$

Rev. 0

STANDARD APPROVAL PAGE

Title:

100-N Area Decision Unit Target Analyse List Development for Soil

Author Name: R. W. Ovine, Integration Project Task Lead

Approval:

J. A. Lurch, Mission Completion

Signature

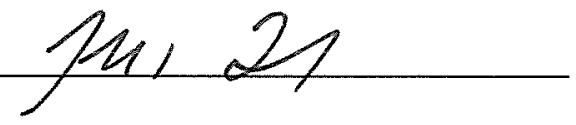

$9 / 14 / 12$

Date

The approval signature on this page indicates that this document has been authorized for information release to the public through appropriate channels. No other forms or signatures are required to document this information release. 
WCH-331

Rev. 0

\title{
River Corridor Closure Contract
}

\section{0-N Area Decision Unit Target Analyte List Development for Soil}

\author{
March 2011
}

Author:

\author{
R. W. Ovink
}




\section{TABLE OF CONTENTS}

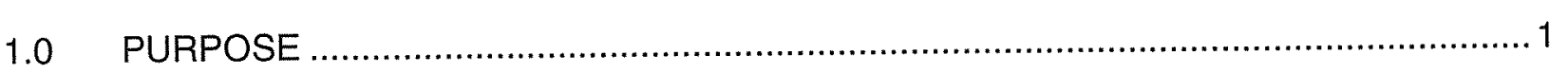

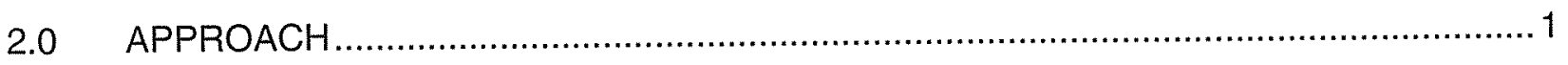

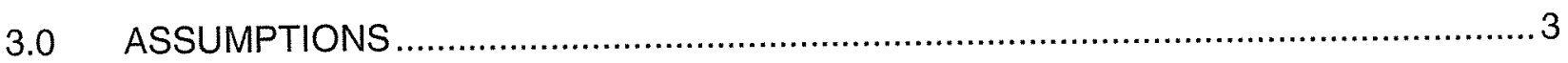

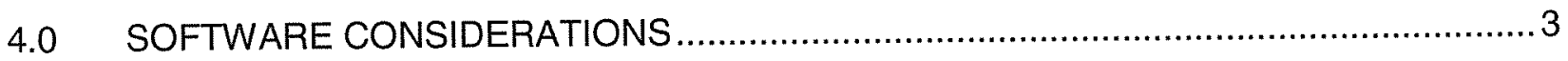

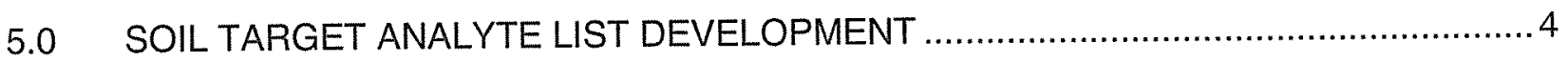

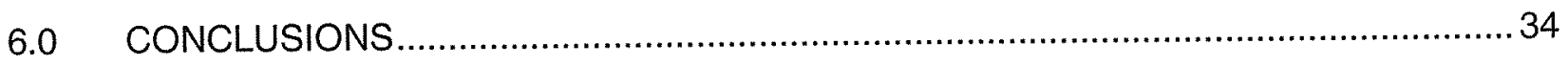

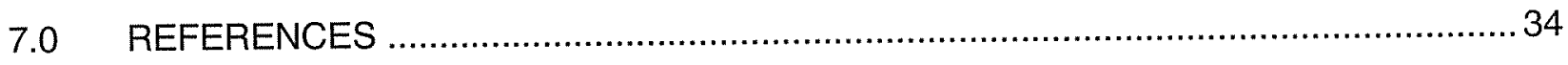

\section{TABLES}

1. Documents Used to Develop the 100-N Area Decision Unit Analyte List.....................4

2. Summary of 100-N Area Target Analytes and References..................................... 5

3. $\quad 100-N$ Area Soil Analytes Excluded from Further Consideration................................... 9

4. Master 100-N Area Target Analytes, Analytical Methods, and Laboratory Detection Limits ..................................................................................................... 10

5. Wells \#1 and \#R1 Target Analytes, Analytical Methods, and Contract-Required Detection Limits..................................................................................... 17

6. Well \#2 Target Analytes, Analytical Methods, and Contract-Required

Detection Limits......................................................................................20

7. Wells \#6 and \#R2 Target Analytes, Analytical Methods, and Contract-Required Detection Limits........................................................................................ 24

8. Wells \#3, \#4, and \#5 Target Analytes, Analytical Methods, and Contract-Required Detection Limits. 


\subsection{PURPOSE}

This report documents the process used to identify source area target analytes in support of the 100-N Area Decision Unit (DU) remedial investigation/feasibility study (RI/FS) addendum to the Integrated 100 Area Remedial Investigation/Feasibility Study (RI/FS) Work Plan (DOE/RL-2008-46). A "target analyte" is defined as a constituent suspected of being site-related that is carried into an investigation plan for characterization through sampling and analysis by approved laboratory methods. Target analytes identified for 100 and 300 Area DUs must support RI/FS nature and extent characterization plus final remedial action decisions for source areas. This report also establishes the analyte exclusion criteria applicable for $100-\mathrm{N}$ use and the analytical methods needed to analyze the target analytes.

\subsection{APPROACH}

The approach for development of vadose zone soil target analytes consists of two steps. The first step is to develop a master list of target analytes. The second step is to develop location specific (e.g., waste site) target analyte lists where additional characterization is proposed.

\section{Step 1 - Decision Unit Master Target Analyte Identification}

A master target analyte list is developed for each Decision Unit using the following approach:

- Review remediation and characterization information (historic and current) and identify appropriate information sources. Sources include:

- Focused feasibility studies (FFS), limited field investigation (LFI) reports

- Interim action records of decision (IARODs)

- Cleanup verification documents (cleanup verification packages [CVPs], remaining sites verification packages [RSVPs])

- Technical baseline reports

- Dangerous waste permit applications

- Databases containing analytical data resulting from these activities (i.e., characterization, remediation, waste management information)

- Other pertinent documents.

- Develop a list of initial target analytes using the reference documents from bullet 1 . 
- Apply the following generally-accepted exclusion criteria that are listed below to the initial set of target analytes to develop the "master" target analyte list.

- Radionuclides with half-lives less than 3 years (and no significant "daughters")

- Naturally occurring radionuclides associated with background radiation (for example, $\mathrm{K}-40$, Th-230, Th-232, and Ra-226)

- Essential nutrients (minerals)

- Analytes that have no toxicity values (based on the most-current version of the Cleanup Levels and Risk Calculation [CLARC] table).

- Compare the master target analyte list for vadose zone soil with the groundwater COPC list developed for the decision unit. Groundwater COPCs not found on the master target analyte list are further evaluated to determine if there is a valid basis for their inclusion.

- Identify appropriate analytical methods for each analyte on the master target analyte list. Determine if the detection limits for each target analyte can achieve the remedial action goals for direct exposure, groundwater protection, and Columbia River protection.

At the conclusion of Step 1, the master decision unit target analyte list is established. This list is comprehensive, including all the contaminants that could potentially be identified as "risk drivers" for waste site remediation within the decision unit under consideration.

\section{Step 2 - Location Specific Target Analyte Identification}

Location specific target analytes are identified using the following approach:

- Identify the contaminants of concern for the specific waste sites where characterization is proposed from the applicable interim action ROD (which reflects information from LFI and technical baseline reports). If the characterization location is not at a waste site, evaluate information from waste sites in the vicinity (where available). Include these analytes on the location specific target analyte list.

- Identify the contaminants of concern for the specific waste site locations from the verification documentation (CVPs or RSVPS). If the characterization location is not at a waste site, evaluate information from waste sites in the vicinity (where available). Include these analytes on the location specific target analyte list.

- Evaluate local groundwater monitoring well data (wells located within waste site "zones of influence"). Determine if groundwater COPCs have been analyzed-for in these local wells.

- If the groundwater COPCs have been analyzed for but not detected, then these analytes are not included on the location specific target analyte list.

- If the groundwater COPCs have been analyzed for and have been detected, then these analytes are included on the location specific target analyte list. 
- If the groundwater COPCs have not been analyzed-for, then an additional evaluation will be performed to determine if there is a data need. If there is a data need, these COPCs are included on the waste-site specific target analyte list.

\subsection{ASSUMPTIONS}

- Historical resources (e.g., LFI, qualitative risk assessment, and CVP/RSVP documents) contain contaminant lists that are comprehensive with respect to characterizing environmental impacts from 100 and 300 Area Hanford Site operations.

- Older analytical data (e.g., pre-Comprehensive Environmental Response, Compensation, and Liability Act of 1980 [CERCLA]) reflect laboratory state-of-the-art procedures. Analytical methods have improved, resulting in lower detection limits for many analytes and better data quality assurance/quality control.

- Characterization activities implemented since initiating remediation under the IARODs may provide additional contaminant information that should be considered during pending RI/FS field investigations.

- Post-remediation characterization and cleanup verification data reflect focused lists of analytes that are unique to each waste site and have been evaluated against IAROD cleanup requirements.

- Examining existing data and waste site process information will be useful in developing laboratory analytical needs for RI/FS characterization tasks.

- Universally-accepted exclusion criteria may be applied to the initial target analyte list to develop a "master" list.

- Additional exclusion criteria (e.g., statistical Hanford Site background comparisons, infrequently detected analytes, and analytes not detected at concentrations/activities exceeding required cleanup levels) may be applied during the RI/FS process as more data become available.

\subsection{SOFTWARE CONSIDERATIONS}

No statistical or algebraic calculations were performed for this activity. The evaluations conducted included analyte comparisons/sorting using Microsoft ${ }^{\oplus}$ Excel $^{\circledR}$.

${ }^{\circledR}$ Microsoft and Excel are registered trademarks of Microsoft Corporation in the United States and/or other countries. 


\subsection{SOIL TARGET ANALYTE LIST DEVELOPMENT}

\section{Step 1 - Decision Unit Master Target Analyte Identification}

1. The documents listed in Table 1 were used to develop the $100-\mathrm{N}$ target analyte list.

Table 1. Documents Used to Develop the 100-N Area Decision Unit Analyte List. (2 Pages)

\begin{tabular}{|c|c|c|}
\hline Reference & Document Number & $\begin{array}{c}\text { Document } \\
\text { Type }\end{array}$ \\
\hline $\begin{array}{l}\text { 1. Cleanup Verification Package/Clean Closure Report for the } \\
\text { Soil Column of the } 120-\mathrm{N}-1 \text { and } 120-\mathrm{N}-2 \text { Dangerous Waste } \\
\text { Treatment and Disposal Sites and the } 100-\mathrm{N}-58 \text { Site }\end{array}$ & CVP-2001-00021 & CVP \\
\hline $\begin{array}{l}\text { 2. Cleanup Verification Package/Clean Closure Report for the } \\
\text { Soil Column of the 116-N-3 Trench, Crib, and } \\
\text { 100-N-63:1 Pipeline }\end{array}$ & CVP-2002-00002 & CVP \\
\hline 3. Cleanup Verification Package for the JA Jones 1 Site & CVP-2001-00019 & CVP \\
\hline $\begin{array}{l}\text { 4. Cleanup Verification Package/Clean Closure Report for the } \\
\text { Soil Column of the 116-N-1 Crib and Trench }\end{array}$ & CVP-2006-00004 & CVP \\
\hline $\begin{array}{l}\text { 5. WA7890008967, Attachment 42, 1324-N Surface } \\
\text { Impoundment, Dangerous Waste Permit Application } \\
\text { Part A Form (August 2005) }\end{array}$ & $\begin{array}{l}\text { WA7890008967, } \\
\text { Attachment } 42\end{array}$ & $\begin{array}{l}\text { Dangerous } \\
\text { Waste } \\
\text { Permit } \\
\text { Application } \\
\end{array}$ \\
\hline $\begin{array}{l}\text { 6. WA7890008967, Attachment 41, 1301-N Liquid Waste } \\
\text { Disposal Facility, Dangerous Waste Permit Application } \\
\text { Part A Form (July 2005) }\end{array}$ & $\begin{array}{l}\text { WA7890008967, } \\
\text { Attachment } 41\end{array}$ & $\begin{array}{l}\text { Dangerous } \\
\text { Waste } \\
\text { Permit } \\
\text { Application } \\
\end{array}$ \\
\hline $\begin{array}{l}\text { 7. WA7890008967, Attachment 42, 1324-NA Percolation Pond, } \\
\text { Dangerous Waste Permit Application Part A Form } \\
\text { (August 2005) }\end{array}$ & $\begin{array}{l}\text { WA7890008967, } \\
\text { Attachment } 42\end{array}$ & $\begin{array}{l}\text { Dangerous } \\
\text { Waste } \\
\text { Permit } \\
\text { Application } \\
\end{array}$ \\
\hline $\begin{array}{l}\text { 8. WA7890008967, Attachment 41, 1325-N Liquid Waste } \\
\text { Disposal Facility, Dangerous Waste Permit Application } \\
\text { Part A Form (August 2005) }\end{array}$ & $\begin{array}{l}\text { WA7890008967, } \\
\text { Attachment } 41\end{array}$ & $\begin{array}{l}\text { Dangerous } \\
\text { Waste } \\
\text { Permit } \\
\text { Application }\end{array}$ \\
\hline $\begin{array}{l}\text { 9. Remedial Design Report/Remedial Action Work Plan for the } \\
\text { 100-N Area (October 2006) }\end{array}$ & DOE/RL-2005-93 & RDR/RAWP \\
\hline $\begin{array}{l}\text { 10. Remedial Design Report/Remedial Action Work Plan for the } \\
\text { 100-NR-1 Treatment, Storage, and disposal Units } \\
\text { (September 2000) }\end{array}$ & DOE/RL-2000-16 & RDR/RAWP \\
\hline $\begin{array}{l}\text { 11. Remedial Design Report/Remedial Action Work Plan for the } \\
\text { 100-NR-2 Operable Unit (June 2002) }\end{array}$ & DOE/RL-2001-27 & RDR/RAWP \\
\hline 12. $100-\mathrm{N}$ Area Technical Baseline Report (July 1994) & WHC-SD-EN-TI-251 & $\begin{array}{l}\text { Technical } \\
\text { Baseline } \\
\text { Report } \\
\end{array}$ \\
\hline 13. 100-NR-1 Qualitative Risk Assessment (March 1995) & BHI-00054 & QRA \\
\hline $\begin{array}{l}\text { 14. 1301-N and 1325-N Liquid Waste disposal Facilities Limited } \\
\text { Field Investigation Report (December 1996) }\end{array}$ & DOE/RL-96-11 & $\begin{array}{l}\text { Limited Field } \\
\text { Investigation }\end{array}$ \\
\hline
\end{tabular}


Table 1. Documents Used to Develop the 100-N Area Decision Unit Analyte List. (2 Pages)

\begin{tabular}{|c|c|c|}
\hline Reference & Document Number & $\begin{array}{l}\text { Document } \\
\text { Type }\end{array}$ \\
\hline $\begin{array}{l}\text { 15. Explanation of Significant Difference for the 100-NR-1 } \\
\text { Operable Unit Treatment, Storage and Disposal Interim Action } \\
\text { Record of Decision and 100-NR-I/100-NR-2 Operable Unit } \\
\text { Interim Action Record of Decision, Hanford Site, } \\
\text { Benton County, Washington. }\end{array}$ & EPA/ESD/R10-03/605 & $\begin{array}{l}\text { Explanation } \\
\text { of } \\
\text { Significant } \\
\text { Difference }\end{array}$ \\
\hline $\begin{array}{l}\text { 16. Interim Remedial Action Record of Decision for the 100-NR-1 } \\
\text { Operable Unit of the Hanford 100-N Area, Hanford Site, } \\
\text { Benton County, Washington }\end{array}$ & $\mathrm{EPA} / 541 / \mathrm{R} 00 / 120$ & IAROD \\
\hline $\begin{array}{l}\text { 17. Interim Action Record of Decision for the 100-NR-1 and } \\
\text { 100-NR-2 Operable Units, Hanford Site, Benton County, } \\
\text { Washington }\end{array}$ & EPA/541/R-99/112 & IAROD \\
\hline $\begin{array}{l}\text { 18. 100-N Area Sampling and Analysis Plan for CERCLA } \\
\text { Waste Sites }\end{array}$ & DOE/RL-2005-92 & SAP \\
\hline $\begin{array}{l}\text { 19. Limited Field Investigation Report for the } 100-N R-2 \\
\text { Operable Unit }\end{array}$ & $\mathrm{DOE} / \mathrm{RL}-93-81$ & $\begin{array}{l}\text { Limited Field } \\
\text { Investigation }\end{array}$ \\
\hline $\begin{array}{l}\text { 20. Limited Field Investigation Report for the 100-NR-1 } \\
\text { Operable Unit (March 1995) }\end{array}$ & DOE/RL-93-80 & $\begin{array}{l}\text { Limited Field } \\
\text { Investigation }\end{array}$ \\
\hline $\begin{array}{l}\text { 21. Cleanup Verification Package for the Hanford Generating Plan } \\
\text { UPR-100-37 Transformer Yard (SWMU \#1), 100-N-51 Oil } \\
\text { Storage Area (SWMU \#2), 185-N Building Drains and Sumps } \\
\text { (SWMU \#3), and 100-N-50 Turbine Oil Filter Unit (SWMU \#4) } \\
\text { (June 2004) }\end{array}$ & $\begin{array}{l}\text { HGP-CVP-SWMUS } 1,2 \\
3, \& 4\end{array}$ & CVP \\
\hline $\begin{array}{l}\text { 22. Cleanup Verification Package for the Hanford Generating } \\
\text { Plant 100- N-4 Tile Field (SWMU \#5); 100-N-1 Settling Pond } \\
\text { (SWMU \#6); 1908-NE Outfall (SWMU \#7); 1716-NE } \\
\text { Maintenance Garage (SWMU \#8) and 100-N-52 Underground } \\
\text { Storage Tank; 100-N-3 Maintenance Garage French Drain, } \\
\text { 100-N-41 Gate House Septic Tank, and 100-N-45 Office } \\
\text { Building Septic Tank (SWMU \#9); 100-N-5 Bone Yard } \\
\text { (SWMU \#10); and 100-N-46 Underground Storage Tank }\end{array}$ & $\begin{array}{l}\text { HGP-CVP-SWMUS } 5,6 \\
7,8,9, \& 10\end{array}$ & CVP \\
\hline $\begin{array}{l}\text { CVP }=\text { cleanup verification package } \\
\text { IAROD }=\text { interim action record of decision } \\
\text { QRA = qualitative risk assessment }\end{array}$ & & \\
\hline
\end{tabular}

2. The initial list of target analytes presented in Table 2 was created from the review and evaluation of the Table 1 documents.

Table 2. Summary of 100-N Area Target Analytes and References. (4 Pages)

\begin{tabular}{|l|l|l|l|l|}
\hline \multicolumn{1}{|c|}{ Analyte } & \multicolumn{2}{c|}{ Reference } & \multicolumn{1}{c|}{ Analyte } & \multicolumn{1}{c|}{ Reference } \\
\hline \multicolumn{5}{|c|}{ Radionuclides } \\
\hline 1. Silver-108m & DOE/RL-2005-92 & 19. Niobium-94 & DOE/RL-2005-92 \\
\hline 2. Americium-241 & CVP-2002-00002 & 20. Plutonium-238 & BHI-00054 \\
\hline 3. Antimony-125 & EPA/541/R-99/112 & 21. Plutonium-239/240 & CVP-2002-00002 \\
\hline 4. Carbon-14 & DOE/RL-2005-92 & 22. Potassium-40 & BHI-00054 \\
\hline
\end{tabular}


Table 2. Summary of 100-N Area Target Analytes and References. (4 Pages)

\begin{tabular}{|c|c|c|c|}
\hline Analyte & Reference & Analyte & Reference \\
\hline 5. Cerium-144 & BHI-00054 & 23. Radium-226 & BHI-00054 \\
\hline 6. Cesium-134 & DOE/RL-2005-93 & 24. Ruthenium-103 & DOE/RL-2005-93 \\
\hline 7. Cesium-137 & CVP-2002-00002 & 25. Ruthenium-106 & DOE/RL-2005-93 \\
\hline 8. Cobalt-60 & CVP-2002-00002 & 26. Sodium-24 & DOE/RL-2005-93 \\
\hline 9. Curium-243 & DOE/RL-2005-92 & 27. Strontium-90 & CVP-2002-00002 \\
\hline 10. Europium-152 & DOE/RL-2005-93 & 28. Technetium-99 & EPA/541/R-99/112 \\
\hline 11. Europium-154 & CVP-2002-00002 & 29. Thorium-228 & BHI-00054 \\
\hline 12. Europium-155 & CVP-2002-00002 & 30. Thorium-230 & DOE/RL-2005-92 \\
\hline 13. lodine-129 & DOE/RL-2005-92 & 31. Thorium-232 & BHI-00054 \\
\hline 14. lodine-131 & DOE/RL-2005-93 & 32. Tritium & CVP-2002-00002 \\
\hline 15. Manganese- 54 & BHI-00054 & 33. Uranium-233/234 & BHI-00054 \\
\hline 16. Manganese-56 & DOE/RL-2005-93 & 34. Uranium-235 & EPA/541/R-99/112 \\
\hline 17. Neptunium-237 & DOE/RL-2005-92 & 35. Uranium-238 & BHI-00054 \\
\hline 18. Nickel-63 & CVP-2002-00002 & & \\
\hline \multicolumn{4}{|c|}{ Nonradionuclides } \\
\hline $\begin{array}{l}\text { 1. 2,4,5-Trichloro- } \\
\text { phenoxyacetic acid }\end{array}$ & DOE/RL-2005-92 & 67. Di-n-butylphthalate & DOE/RL-2005-92 \\
\hline 2. Acetone & DOE/RL-2005-92 & 68. Di-n-octylphthalate & DOE/RL-2005-92 \\
\hline 3. Acenaphthene & DOE/RL-2005-92 & 69. Di-nitro-2-methylphenol; 4,6- & DOE/RL-2005-92 \\
\hline 4. Acenaphthylene & DOE/RL-2005-92 & 70. Dinitrophenol; 2,4- & DOE/RL-2005-92 \\
\hline 5. Aldrin & DOE/RL-2005-92 & 71. Dinitrotoluene; 2,4- & DOE/RL-2005-92 \\
\hline $\begin{array}{l}\text { 6. Aluminum sulfate } \\
\text { (Aluminum) }\end{array}$ & DOE/RL-2005-93 & 72. Dinitrotoluene; $2,6-$ & DOE/RL-2005-92 \\
\hline 7. Anthracene & DOE/RL-2005-93 & 73. Dinoseb (DNBP) & DOE/RL-2005-92 \\
\hline 8. Antimony & EPA/541/R-99/112 & 74. Endosulfan (I, II, sulfate) & DOE/RL-2005-92 \\
\hline 9. Arsenic & DOE/RL-2005-93 & $\begin{array}{l}\text { 75. Endrin (and ketone, } \\
\text { aldehyde) }\end{array}$ & DOE/RL-2005-92 \\
\hline 10. Asbestos & DOE/RL-2005-93 & 76. Fluoranthene & DOE/RL-2005-93 \\
\hline 11. Barium & CVP-2001-00021 & 77. Fluorene & DOE/RL-2005-93 \\
\hline 12. Benzene & DOE/RL-93-80 & 78. Fluoride & DOE/RL-93-81 \\
\hline $\begin{array}{l}\text { 13. Benzo(a)anthrax- } \\
\text { cene }\end{array}$ & DOE/RL-2005-93 & 79. Heptachlor & DOE/RL-2005-92 \\
\hline 14. Benzo(a)pyrene & DOE/RL-2005-93 & 80. Heptachlor epoxide & DOE/RL-2005-92 \\
\hline $\begin{array}{l}\text { 15. Benzo(b)fluor- } \\
\text { anthene }\end{array}$ & DOE/RL-2005-93 & 81. Hexachlorobenzene & DOE/RL-2005-92 \\
\hline $\begin{array}{l}\text { 16. Benzo(ghi)- } \\
\text { perylene }\end{array}$ & DOE/RL-2005-93 & 82. Hexachlorobutadiene & DOE/RL-2005-92 \\
\hline
\end{tabular}


Table 2. Summary of 100-N Area Target Analytes and References. (4 Pages)

\begin{tabular}{|c|c|c|c|}
\hline Analyte & Reference & Analyte & Reference \\
\hline $\begin{array}{l}\text { 17. Benzo(k)fluor- } \\
\text { anthene }\end{array}$ & DOE/RL-2005-93 & 83. Hexachlorocyclopentadiene & DOE/RL-2005-92 \\
\hline 18. Beryllium & DOE/RL-2005-93 & 84. Hexachloroethane & DOE/RL-2005-92 \\
\hline 19. BHC; alpha & DOE/RL-2005-92 & 85. Indeno(1,2,3-cd)pyrene & DOE/RL-2005-93 \\
\hline 20. BHC; beta & DOE/RL-2005-92 & 86. Iron & DOE/RL-93-81 \\
\hline 21. BHC; delta & DOE/RL-2005-92 & 87. Isophorone & DOE/RL-2005-92 \\
\hline $\begin{array}{l}\text { 22. BHC; gamma } \\
\text { (lindane) }\end{array}$ & DOE/RL-2005-92 & 88. Lead & CVP-2001-00021 \\
\hline $\begin{array}{l}\text { 23. Bis(2-chloro-1- } \\
\text { methylethyl) ether }\end{array}$ & DOE/RL-2005-92 & 89. Lithium & DOE/RL-2005-92 \\
\hline $\begin{array}{l}\text { 24. Bis(2-chloroethoxy) } \\
\text { methane }\end{array}$ & DOE/RL-2005-92 & 90. Manganese & DOE/RL-2005-93 \\
\hline $\begin{array}{l}\text { 25. Bis(2-ethylhexyl) } \\
\text { phthalate }\end{array}$ & DOE/RL-93-80 & 91. Mercury & CVP-2001-00021 \\
\hline 26. Boron & DOE/RL-2005-93 & 92. Methoxychlor & DOE/RL-2005-92 \\
\hline $\begin{array}{l}\text { 27. Bromophenyl- } \\
\text { phenyl ether; } 4-\end{array}$ & DOE/RL-2005-92 & 93. Methylene chloride & DOE/RL-2005-92 \\
\hline 28. Butanone; 2- & DOE/RL-2005-92 & 94. Methylnaphthalene; 2- & DOE/RL-2005-92 \\
\hline $\begin{array}{l}\text { 29. Butylbenzyl- } \\
\text { phthalate }\end{array}$ & DOE/RL-2005-92 & 95. Methylphenol; 2- (cresol; o-) & DOE/RL-2005-92 \\
\hline 30. Cadmium & CVP-2001-00019 & 96. Methylphenol; 4- (cresol; p-) & DOE/RL-2005-92 \\
\hline 31. Carbon disulfide & $\mathrm{DOE} / \mathrm{RL}-93-80$ & 97. Molybdenum & DOE/RL-2005-92 \\
\hline $\begin{array}{l}\text { 32. Carbon } \\
\text { tetrachloride }\end{array}$ & EPA/541/R-99/112 & 98. Naphthalene & DOE/RL-2005-92 \\
\hline 33. Carbazole & DOE/RL-2005-92 & 99. Nickel & CVP-2001-00021 \\
\hline $\begin{array}{l}\text { 34. Chlordane (alpha, } \\
\text { gamma) }\end{array}$ & DOE/RL-2005-92 & 100. Nitrate & CVP-2002-00002 \\
\hline 35. Chlorine & DOE/RL-2005-93 & 101. Nitroaniline; 2- & DOE/RL-2005-92 \\
\hline $\begin{array}{l}\text { 36. Chloro-3- } \\
\text { methylphenol;4- }\end{array}$ & DOE/RL-2005-92 & 102. Nitroaniline; 3- & DOE/RL-2005-92 \\
\hline 37. Chloroanilene;4- & DOE/RL-2005-92 & 103. Nitroaniline; 4- & DOE/RL-2005-92 \\
\hline $\begin{array}{l}\text { 38. Chloronaphtha- } \\
\text { lene;2- }\end{array}$ & DOE/RL-2005-92 & 104. Nitrobenzene & DOE/RL-2005-92 \\
\hline 39. Chlorophenol;2- & DOE/RL-2005-92 & 105. Nitrophenol; 2- & DOE/RL-2005-92 \\
\hline $\begin{array}{l}\text { 40. Chlorophenyl- } \\
\text { phenyl ether;4- }\end{array}$ & DOE/RL-2005-92 & 106. Nitrophenol; 4- & DOE/RL-2005-92 \\
\hline 41. Chloroform & EPA/541/R-99/112 & 107. Nitroso-di-n-propylamine; N- & DOE/RL-2005-92 \\
\hline $\begin{array}{l}\text { 42. Chromium } \\
\text { (hexavalent) }\end{array}$ & DOE/RL-2005-93 & 108. Nitrosodiphenylamine; N- & DOE/RL-2005-92 \\
\hline 43. Chromium (total) & CVP-2001-00021 & 109. PCBs & DOE/RL-2005-93 \\
\hline 44. Chrysene & DOE/RL-2005-93 & 110. Pentachlorophenol & DOE/RL-2005-92 \\
\hline 45. Cobalt & DOE/RL-2005-93 & 111. Phenanthrene & DOE/RL-2005-93 \\
\hline
\end{tabular}


Table 2. Summary of 100-N Area Target Analytes and References. (4 Pages)

\begin{tabular}{|c|c|c|c|}
\hline Analyte & Reference & Analyte & Reference \\
\hline 46. Copper & CVP-2001-00021 & 112. Phenol & DOE/RL-2005-92 \\
\hline 47. Cyanide & DOE/RL-2005-92 & 113. Phosphoric acid & DOE/RL-2005-93 \\
\hline 48. Dalapon & DOE/RL-2005-92 & 114. Pyrene & DOE/RL-2005-93 \\
\hline 49. D B 2;4- & DOE/RL-2005-92 & 115. Selenium & EPA/541/R-99/112 \\
\hline 50. DDD; 4,4'- & DOE/RL-2005-92 & 116. Silver & DOE/RL-2005-92 \\
\hline 51. DDE; 4,4'- & DOE/RL-2005-92 & 117. Silvex (TP; $2,4,5-)$ & DOE/RL-2005-92 \\
\hline 52. DDT; 4,4'- & DOE/RL-2005-92 & 118. Sodium dichromate & DOE/RL-2005-93 \\
\hline $\begin{array}{l}\text { 53. Dibenz(a,h)- } \\
\text { anthracene }\end{array}$ & DOE/RL-2005-93 & 119. Sodium hydroxide & DOE/RL-2005-93 \\
\hline 54. Dibenzofuran & DOE/RL-2005-92 & 120. Sulfate & CVP-2001-00021 \\
\hline 55. Dicamba & DOE/RL-2005-92 & 121. Sulfide & DOE/RL-2005-92 \\
\hline $\begin{array}{l}\text { 56. Dichlorobenzene; } \\
1,2 \text { - }\end{array}$ & DOE/RL-2005-92 & 122. Tetrachloroethene & EPA/541/R-99/112 \\
\hline $\begin{array}{l}\text { 57. Dichlorobenzene; } \\
1,3-\end{array}$ & DOE/RL-2005-92 & 123. Tributyl phosphate & DOE/RL-2005-92 \\
\hline $\begin{array}{l}\text { 58. Dichlorobenzene; } \\
\text { 1,4- }\end{array}$ & DOE/RL-2005-92 & 124. Trichlorobenzene; $1,2,4-$ & DOE/RL-2005-92 \\
\hline $\begin{array}{l}\text { 59. Dichlorobenzidine; } \\
3,3^{\prime}-\end{array}$ & DOE/RL-2005-92 & 125. Trichloroethane; $1,1,1-$ & DOE/RL-93-80 \\
\hline $\begin{array}{l}\text { 60. Dichlorophenol; } \\
\text { 2,4- }\end{array}$ & DOE/RL-2005-92 & 126. Trichlorophenol; 2,4,5- & DOE/RL-2005-92 \\
\hline $\begin{array}{l}\text { 61. Dichlorophenoxy- } \\
\text { acetic acid; } 2,4-\end{array}$ & DOE/RL-2005-92 & 127. Trichlorophenol; 2,4,6- & DOE/RL-2005-92 \\
\hline 62. Dichlorprop & DOE/RL-2005-92 & 128. Toluene & DOE/RL-2005-92 \\
\hline 63. Dieldrin & DOE/RL-2005-92 & 129. Toxaphene & DOE/RL-2005-92 \\
\hline 64. Diethylphthalate & DOE/RL-2005-92 & 130. TPH/diesel and motor oil & DOE/RL-2005-93 \\
\hline 65. Dimethylphthalate & DOE/RL-2005-92 & 131. Vanadium & DOE/RL-2005-93 \\
\hline $\begin{array}{l}\text { 66. Dimethylphenol; } \\
2,4-\end{array}$ & DOE/RL-2005-92 & 132. Zinc & CVP-2001-00021 \\
\hline
\end{tabular}

$\mathrm{PCB}=$ polychlorinated biphenyl

$\mathrm{TPH}=$ total petroleum hydrocarbons

3. The generally accepted exclusion criteria that follow were applied to the initial soil target analyte list to identify the excluded analytes listed in Table 3 and to develop the master target analyte list presented in Table 4.

- Radionuclides with half-lives less than 3 years (and no significant "daughters")

- Naturally occurring radionuclides associated with background radiation

- Essential nutrients (minerals)

- Analytes that have no toxicity values (per the most current CLARC table). 
Table 3. 100-N Area Soil Analytes Excluded from Further Consideration.

\begin{tabular}{|c|c|c|}
\hline Analyte & Exclusion Rationale & $\begin{array}{l}\text { Daughters } \\
\text { (half-life) }\end{array}$ \\
\hline \multicolumn{3}{|c|}{ Radionuclides } \\
\hline Antimony-125 & Half-life less than 3 years $(2.76 \mathrm{y})$ & Te-125m (58d) Te-125 (stable) \\
\hline Cerium-144 & Half-life less than 3 years (284.6d) & Ba-134 (stable) \\
\hline Cesium-134 & Half-life less than 3 years $(2.065 \mathrm{y})$ & Ba-134 (stable) \\
\hline lodine-131 & Half-life less than 3 years $(8.02 \mathrm{~d})$ & $\begin{array}{l}\text { Xe-131m (11.8d) Xe-131 } \\
\text { (stable) }\end{array}$ \\
\hline Manganese- 54 & Half-life less than 3 years $(612.2 d)$ & Fe-54 (stable) \\
\hline Ruthenium-103 & Half-life less than 3 years $(39.27 \mathrm{~d})$ & $\begin{array}{l}\text { Rh-103m (56.12m), Rh-103 } \\
\text { (stable) }\end{array}$ \\
\hline Ruthenium-106 & Half-life less than 3 years $(1.020 y)$ & Rh-106 (29.9s), Pd-106 (stable) \\
\hline Manganese-56 & Half-life less than 3 years (2.5h) & Fe-56 (stable) \\
\hline Potassium -40 & Naturally occurring background radiation & 1.28 E9 years \\
\hline Radium-226 & $\begin{array}{l}\text { Only potential source from naturally occurring } \\
\text { background radiation (insufficient in-growth time } \\
\text { for Hanford introduced } U \text { as decay daughter of } \\
\text { U-234/Th-230) }\end{array}$ & 1.6 E3 years \\
\hline Sodium-24 & Half-life less than 3 years $(15 \mathrm{~h})$ & Mg-24 (stable) \\
\hline Thorium-228 & $\begin{array}{l}\text { Decay daughter of Th-232/Ra-228. Will be in } \\
\text { equilibrium with parent }\end{array}$ & 1.91 years \\
\hline Thorium-230 & $\begin{array}{l}\text { Only potential source from naturally occurring } \\
\text { background radiation (insufficient in-growth time } \\
\text { for Hanford introduced } U \text { as decay daughter of } \\
U-234 \text { ) }\end{array}$ & $7.7 \mathrm{E} 4$ years \\
\hline Thorium-232 & Naturally occurring background radiation & 1.4 E10 years \\
\hline \multicolumn{3}{|c|}{ Nonradionuclides } \\
\hline Acetone & Laboratory contaminant & None \\
\hline $\begin{array}{l}\text { Bis(2-ethylhexyl) } \\
\text { phthalate }\end{array}$ & Laboratory contaminant & None \\
\hline Fluoride & Essential nutrient (minerals) & None \\
\hline Iron & Essential nutrient & None \\
\hline Methylene chloride & Laboratory contaminant & None \\
\hline Phenol & $\begin{array}{l}\text { Naturally occurring, readily biodegradable } \\
\text { organic compound }\end{array}$ & None \\
\hline Sulfate & No soil toxicity information available & None \\
\hline Sulfide & No soil toxicity information available & None \\
\hline
\end{tabular}


Table 4. Master 100-N Area Target Analytes, Analytical Methods, and Laboratory Detection Limits. (7 Pages)

\begin{tabular}{|c|c|c|c|c|c|}
\hline \multirow[b]{2}{*}{ Target Analyte } & \multirow{2}{*}{$\begin{array}{c}\text { Practical } \\
\text { Quantitation } \\
\text { Limits }^{a}\end{array}$} & \multicolumn{3}{|c|}{ Preliminary Cleanup Goals ${ }^{a, b}$} & \multirow{2}{*}{$\begin{array}{l}\text { Analytical } \\
\text { Methods }\end{array}$} \\
\hline & & $\begin{array}{c}\text { Direct } \\
\text { Exposure }\end{array}$ & $\begin{array}{l}\text { Groundwater } \\
\text { Protection }\end{array}$ & $\begin{array}{c}\text { River } \\
\text { Protection }\end{array}$ & \\
\hline \multicolumn{6}{|c|}{ Radionuclides } \\
\hline Americium-241 ${ }^{c}$ & 1 & 31.1 & NV & NV & $\begin{array}{l}\mathrm{Am}-241 / \\
\mathrm{Cm}-244 \text { AEA }\end{array}$ \\
\hline Curium-243 & 1 & 22.1 & NV & NV & $\begin{array}{l}\mathrm{Am}-241 / \\
\mathrm{Cm}-244 \mathrm{AEA}\end{array}$ \\
\hline Cesium-137 & 0.1 & 6.2 & 1,465 & 2,930 & \multirow{7}{*}{ GEA } \\
\hline Cobalt- 60 & 0.05 & 1.4 & 13,900 & 27,800 & \\
\hline Europium-152 & 0.1 & 3.3 & NV & NV & \\
\hline Europium-154 & 0.1 & 3.0 & NV & NV & \\
\hline Europium-155 & 0.1 & 125 & NV & NV & \\
\hline Niobium-94 & 0.2 & 2.43 & NV & NV & \\
\hline Silver- $108 \mathrm{~m}$ & 0.2 & 2.38 & NV & NV & \\
\hline Strontium-90 & 1 & 4.5 & 27.6 & 55.2 & $\begin{array}{l}\text { Gas flow } \\
\text { proportional } \\
\text { counting }\end{array}$ \\
\hline lodine-129 & 2 & $2^{b}$ & $2^{b}$ & $2^{b}$ & $\begin{array}{l}\text { Low energy } \\
\text { GEA }\end{array}$ \\
\hline Plutonium-238 & 1 & 35.1 & NV & NV & \multirow{2}{*}{$\begin{array}{l}\text { Isotopic } \mathrm{Pu} \\
\mathrm{AEA}\end{array}$} \\
\hline Plutonium-239/240 & 1 & 33.9 & NV & NV & \\
\hline Uranium-233/234 & 1 & $1.1^{b}$ & $1.1^{b}$ & $1.1^{b}$ & \multirow{3}{*}{$\begin{array}{l}\text { Isotopic U } \\
\text { AEA }\end{array}$} \\
\hline Uranium-235 & 1 & 0.61 & $0.185^{d}$ & $0.185^{d}$ & \\
\hline Uranium-238 & 1 & $1.1^{b}$ & $1.1^{b}$ & $1.1^{b}$ & \\
\hline Carbon-14 & 2 & 8.7 & NV & NV & \multirow{4}{*}{$\begin{array}{l}\text { Liquid } \\
\text { scintillation } \\
\text { counting }\end{array}$} \\
\hline Nickel-63 & 30 & 4,026 & 83 & 166 & \\
\hline Technetium-99 & 0.25 & 5.7 & 0.46 & 0.46 & \\
\hline Tritium & 10 & 510 & 12.6 & 25.2 & \\
\hline Neptunium-237 & 1 & 2.44 & $0.9^{d}$ & $1.80^{d}$ & $\begin{array}{l}\text { Np-237 } \\
\text { AEA }\end{array}$ \\
\hline \multicolumn{6}{|c|}{ Nonradionuclides } \\
\hline Chloride & 2 & NV & 25,000 & NA & \multirow{5}{*}{$\begin{array}{l}\text { EPA } 300.0 \\
\text { (anions } \\
\text { by } I C \text { ) }\end{array}$} \\
\hline Fluoride & 5 & 4,800 & 2,800 & 2,890 & \\
\hline Nitrate (as nitrogen) & 2.5 & 128,000 & 40 & 40 & \\
\hline Nitrite (as nitrogen) & 2.5 & 8,000 & 40 & 4 & \\
\hline Sulfate & 5 & NV & 1,030 & 1,030 & \\
\hline
\end{tabular}


Table 4. Master 100-N Area Target Analytes, Analytical Methods, and Laboratory Detection Limits. (7 Pages)

\begin{tabular}{|c|c|c|c|c|c|}
\hline \multirow[b]{2}{*}{ Target Analyte } & \multirow{2}{*}{$\begin{array}{c}\text { Practical } \\
\text { Quantitation } \\
\text { Limits }^{a}\end{array}$} & \multicolumn{3}{|c|}{ Preliminary Cleanup Goals ${ }^{a, b}$} & \multirow{2}{*}{$\begin{array}{l}\text { Analytical } \\
\text { Methods }\end{array}$} \\
\hline & & $\begin{array}{c}\text { Direct } \\
\text { Exposure }\end{array}$ & $\begin{array}{c}\text { Groundwater } \\
\text { Protection }\end{array}$ & $\begin{array}{c}\text { River } \\
\text { Protection }\end{array}$ & \\
\hline Aluminum & 5 & 80,000 & 480,000 & 480,000 & \multirow{20}{*}{$\begin{array}{l}\text { EPA } 6010 \text { or } \\
200.8 \text { (ICP or } \\
\text { ICP/MS) }\end{array}$} \\
\hline Antimony & 6 & 32 & 5.4 & 12.7 & \\
\hline Arsenic & 10 & $\mathrm{TBD}^{\mathrm{b}}$ & $\mathrm{TBD}^{\mathrm{b}}$ & $\mathrm{TBD}^{\mathrm{b}}$ & \\
\hline Barium & 2 & 16,000 & 1,650 & 1,600 & \\
\hline Beryllium & 0.5 & 160 & 63.2 & 63 & \\
\hline Boron & 2 & 16,000 & 210 & NV & \\
\hline Cadmium & 0.5 & 80 & 0.69 & $0.125^{d}$ & \\
\hline Chromium (total) & 1 & 120,000 & 2,000 & 1,300 & \\
\hline Cobalt & 2 & 24 & 15.7 & NV & \\
\hline Copper & 1 & 3,200 & 284 & 575 & \\
\hline Lead & 5 & 250 & 3,000 & 420 & \\
\hline Lithium & 2.5 & 160 & 192 & NV & \\
\hline Manganese & 5 & 3,760 & $512^{b}$ & $256^{\mathrm{b}}$ & \\
\hline Molybdenum & 2 & 400 & 32 & NV & \\
\hline Nickel & 4 & 1,600 & 130 & 179 & \\
\hline Selenium & 10 & 400 & $5.2^{d}$ & $0.52^{d}$ & \\
\hline Silver & 1 & 400 & 13.6 & 0.442 & \\
\hline Thallium & 5 & 5.6 & 1.59 & 4.46 & \\
\hline Vanadium & 2.5 & 560 & 2,240 & NV & \\
\hline Zinc & 1 & 24,000 & 5,970 & 226 & \\
\hline Chromium (hexavalent) & 0.5 & TBD & TBD & TBD & $\begin{array}{l}\text { EPA } 7196 \\
\text { (hexavalent } \\
\text { chromium) }\end{array}$ \\
\hline Mercury & 0.2 & 24 & 2.09 & $0.33^{b}$ & $\begin{array}{l}\text { EPA 7471 } \\
\text { (Hg cold } \\
\text { vapor) or } \\
200.8 \\
\text { (ICP/MS) } \\
\end{array}$ \\
\hline Ethylene glycol & 5.0 & 160,000 & 64.3 & NV & EPA 8015 \\
\hline Propylene glycol & 5 & $1,600,000$ & NV & NV & EPA 8015 \\
\hline Methanol & 1.0 & 4,000 & 16.1 & NV & EPA $8015 M$ \\
\hline
\end{tabular}


Table 4. Master 100-N Area Target Analytes, Analytical Methods, and Laboratory Detection Limits. (7 Pages)

\begin{tabular}{|c|c|c|c|c|c|}
\hline \multirow[b]{2}{*}{ Target Analyte } & \multirow{2}{*}{$\begin{array}{c}\text { Practical } \\
\text { Quantitation } \\
\text { Limits }^{a}\end{array}$} & \multicolumn{3}{|c|}{ Preliminary Cleanup Goals ${ }^{a, b}$} & \multirow{2}{*}{$\begin{array}{l}\text { Analytica } \\
\text { Methods }\end{array}$} \\
\hline & & $\begin{array}{c}\text { Direct } \\
\text { Exposure }\end{array}$ & $\begin{array}{c}\text { Groundwater } \\
\text { Protection }\end{array}$ & $\begin{array}{c}\text { River } \\
\text { Protection } \\
\end{array}$ & \\
\hline Aldrin & 0.00165 & 0.0588 & 0.00504 & $0.0008^{d}$ & \multirow{20}{*}{$\begin{array}{l}\text { EPA } 8081 \\
\text { (pesticide) }\end{array}$} \\
\hline BHC; alpha & 0.00165 & 0.159 & $0.0005^{d}$ & $0.00031^{d}$ & \\
\hline $\mathrm{BHC}$; beta & 0.00165 & 0.556 & 0.00227 & 0.00259 & \\
\hline $\mathrm{BHC}$; delta & 0.00165 & NV & NV & NV & \\
\hline BHC; gamma (lindane) & 0.00165 & 0.769 & 0.00209 & 0.00119 & \\
\hline Chlordane & 0.0165 & 2.86 & 2.06 & $0.00055^{d}$ & \\
\hline DDD; 4,4'- & 0.0033 & 4.17 & 0.335 & $0.00024^{d}$ & \\
\hline DDE; 4,4'- & 0.0033 & 2.94 & 0.446 & $0.006^{d}$ & \\
\hline DDT; 4,4'- & 0.0033 & 2.94 & 3.49 & 0.00493 & \\
\hline Dieldrin & 0.0033 & 0.0625 & $0.0028^{d}$ & $0.00004^{d}$ & \\
\hline Endosulfan I & 0.00165 & 480 & 4.3 & 0.0417 & \\
\hline Endosulfan II & 0.0033 & 480 & 4.3 & 0.0417 & \\
\hline Endosulfan sulfate & 0.0033 & 480 & 4.3 & 0.0417 & \\
\hline Endrin & 0.0033 & 24 & 0.44 & 0.168 & \\
\hline Endrin aldehyde & 0.0033 & 24 & 0.44 & 0.168 & \\
\hline Endrin ketone & 0.0033 & 24 & 0.44 & 0.168 & \\
\hline Heptachlor & 0.00165 & 0.222 & 0.0370 & $0.000025^{d}$ & \\
\hline Heptachlor epoxide & 0.00165 & 0.11 & 0.008 & $0.0001^{d}$ & \\
\hline Methoxychlor & 0.0165 & 400 & 64.2 & 13.4 & \\
\hline Toxaphene & 0.165 & 0.909 & 0.153 & 0.0005 & \\
\hline Aroclor-1016 (PCB) & 0.0165 & 0.5 & 0.094 & $0.000224^{d}$ & \multirow{8}{*}{$\begin{array}{l}\text { EPA } 8082 \\
\text { (PCBs) }\end{array}$} \\
\hline Aroclor-1221 (PCB) & 0.0165 & 0.5 & $0.00920^{d}$ & $0.0000219^{d}$ & \\
\hline Aroclor-1232 (PCB) & 0.0165 & 0.5 & $0.00920^{d}$ & $0.0000219^{d}$ & \\
\hline Aroclor-1242 (PCB) & 0.0165 & 0.5 & 0.0394 & $0.0000935^{d}$ & \\
\hline Aroclor-1248 (PCB) & 0.0165 & 0.5 & 0.0386 & $0.0000935^{d}$ & \\
\hline Aroclor-1254 (PCB) & 0.0165 & 0.5 & 0.0664 & $0.000158^{d}$ & \\
\hline Aroclor-1260 (PCB) & 0.0165 & 0.5 & 0.721 & $0.00171^{d}$ & \\
\hline Aroclor-1262 (PCB) & 0.0165 & NV & NV & NV & \\
\hline
\end{tabular}


Table 4. Master 100-N Area Target Analytes, Analytical Methods, and Laboratory Detection Limits. (7 Pages)

\begin{tabular}{|c|c|c|c|c|c|}
\hline \multirow[b]{2}{*}{ Target Analyte } & \multirow{2}{*}{$\begin{array}{c}\text { Practical } \\
\text { Quantitation } \\
\text { Limits }^{a}\end{array}$} & \multicolumn{3}{|c|}{ Preliminary Cleanup Goals ${ }^{a, b}$} & \multirow{2}{*}{$\begin{array}{l}\text { Analytical } \\
\text { Methods }\end{array}$} \\
\hline & & $\begin{array}{c}\text { Direct } \\
\text { Exposure }\end{array}$ & $\begin{array}{c}\text { Groundwater } \\
\text { Protection }\end{array}$ & $\begin{array}{c}\text { River } \\
\text { Protection }\end{array}$ & \\
\hline Dalapon & 0.1 & 2,400 & 0.811 & 0.81 & \multirow{8}{*}{$\begin{array}{l}\text { EPA } 8151 \\
\text { (herbicides) }\end{array}$} \\
\hline $\mathrm{DB} ; 2,4-$ & 0.1 & 640 & 0.768 & NV & \\
\hline Dicamba & 0.1 & 2,400 & 2.2 & 2.20 & \\
\hline $\begin{array}{l}\text { Dichlorophenoxyacetic } \\
\text { acid; } 2,4-\end{array}$ & 0.4 & 800 & $0.321^{d}$ & 0.321 & \\
\hline Dichlorprop & 0.1 & 640 & 0.321 & 0.321 & \\
\hline $\begin{array}{l}\text { Dinoseb (DNBP) }\{2-\mathrm{sec}- \\
\text { butyl-4,6-dinitrophenol }\}\end{array}$ & 0.012 & 80 & 0.0524 & 1.05 & \\
\hline TP; 2,4,5- (Silvex) & 0.02 & 640 & 0.28 & 0.28 & \\
\hline $\begin{array}{l}\text { Trichlorophenoxyacetic } \\
\text { acid; } 2,4,5 \text { - }\end{array}$ & 0.02 & 800 & 0.761 & 0.79 & \\
\hline Acetone & 0.02 & 72,000 & 28.9 & NV & \multirow{20}{*}{$\begin{array}{l}\text { EPA } 8260 \\
\text { (VOCs) }\end{array}$} \\
\hline Benzene & 0.005 & 18.2 & 0.004 & 0.007 & \\
\hline Butanol; 1- & 0.1 & 8,000 & 3.31 & 6.6 & \\
\hline Butanone; 2- & 0.01 & 48,000 & 19.6 & NV & \\
\hline Carbon disulfide & 0.005 & 800 & 5.65 & 12.4 & \\
\hline Carbon tetrachloride & 0.005 & 7.69 & 0.031 & $0.0023^{d}$ & \\
\hline Chloroform & 0.005 & 164. & 0.038 & 0.0304 & \\
\hline Cyclohexanone & 1 & 400,000 & 172 & NV & \\
\hline Ethyl acetate & 5 & NV & 29.8 & NV & \\
\hline Ethyl ether & 0.005 & 16,000 & 6.68 & NV & \\
\hline Ethylbenzene & 0.005 & 8,000 & 6.05 & 26.8 & \\
\hline Methyl isobutyl ketone & 0.01 & 6,400 & 2.71 & NV & \\
\hline Methylene chloride & 0.005 & 133 & 0.218 & 0.0205 & \\
\hline Tetrachloroethene & 0.005 & 1.85 & 0.008 & 0.004 & \\
\hline Toluene & 0.005 & 6,400 & 4.65 & 49.5 & \\
\hline Trichlorobenzene; $1,2,4-$ & 0.01 & 800 & 2.98 & 1.31 & \\
\hline Trichloroethane; 1,1,1- & 0.005 & NV & 1.58 & 1.59 & \\
\hline Trichloroethene & 0.005 & 11.2 & 0.00323 & 0.0178 & \\
\hline Vinyl chloride & 0.005 & 240 & 0.00184 & 0.0126 & \\
\hline Xylenes (total) & 0.01 & 16,000 & 3.09 & 5.15 & \\
\hline
\end{tabular}


Table 4. Master 100-N Area Target Analytes, Analytical Methods, and Laboratory Detection Limits. (7 Pages)

\begin{tabular}{|c|c|c|c|c|c|}
\hline \multirow[b]{2}{*}{ Target Analyte } & \multirow{2}{*}{$\begin{array}{c}\text { Practical } \\
\text { Quantitation } \\
\text { Limits }^{a}\end{array}$} & \multicolumn{3}{|c|}{ Preliminary Cleanup Goals $^{a, b}$} & \multirow{2}{*}{$\begin{array}{l}\text { Analytica } \\
\text { Methods }\end{array}$} \\
\hline & & $\begin{array}{c}\text { Direct } \\
\text { Exposure }\end{array}$ & $\begin{array}{c}\text { Groundwater } \\
\text { Protection }\end{array}$ & $\begin{array}{c}\text { River } \\
\text { Protection }\end{array}$ & \\
\hline Tributyl phosphate & 3.3 & 185 & $0.677^{d}$ & NV & \multirow{29}{*}{$\begin{array}{l}\text { EPA } 8270 \\
\text { (SVOCs) }\end{array}$} \\
\hline $\begin{array}{l}\text { Bis(2-chloro-1-methylethyl) } \\
\text { ether }\end{array}$ & 0.33 & 14.3 & NV & 0.180 & \\
\hline $\begin{array}{l}\text { Bis(2-chloroethoxy) } \\
\text { methane }\end{array}$ & 0.33 & 0.909 & NV & NV & \\
\hline Bis(2-ethylhexyl)phthalate & 0.33 & 71.4 & 13.9 & 4.01 & \\
\hline $\begin{array}{l}\text { Bromophenylphenyl ether; } \\
\text { 4- }\end{array}$ & 0.33 & NV & NV & NV & \\
\hline Butylbenzylphthalate & 0.33 & 16,000 & 893 & 349 & \\
\hline Carbazole & 0.33 & 50 & $0.314^{d}$ & NV & \\
\hline Chloro-3-methylphenol;4- & 0.33 & 4,000 & NV & NV & \\
\hline $\begin{array}{l}\text { Chlorophenylphenyl } \\
\text { ether;4- }\end{array}$ & 0.33 & 4,000 & NV & NV & \\
\hline Dibenzofuran & 0.33 & 160 & 7.36 & NV & \\
\hline Diethylphthalate & 0.33 & 64,000 & 72.2 & 130 & \\
\hline Di-n-butylphthalate & 0.33 & 8,000 & 56.5 & 95.5 & \\
\hline Di-n-octylphthalate & 0.33 & 1,600 & 532,000 & NV & \\
\hline Isophorone & 0.33 & 1,050 & $0.228^{d}$ & 7.7 & \\
\hline Methylnaphthalene; 2- & 0.33 & 320 & 2.03 & 2.04 & \\
\hline Methylphenol; 4-(cresol; p-) & 0.33 & 400 & 0.507 & 0.505 & \\
\hline Chloroaniline;4- & 0.33 & 320 & $0.264^{d}$ & NV & \\
\hline Chloronaphthalene;2- & 0.33 & 6,400 & 40.7 & 56.5 & \\
\hline Chlorophenol;2- & 0.33 & 400 & 0.472 & 2.28 & \\
\hline Dichlorobenzene; 1,2- & 0.33 & 7,200 & 7.03 & 31.7 & \\
\hline Dichlorobenzene; 1,3- & 0.33 & 2,400 & 3.09 & 5.15 & \\
\hline Dichlorobenzene; 1,4- & 0.33 & 41.7 & $0.030^{d}$ & $0.080^{d}$ & \\
\hline Dichlorobenzidine; $3,3^{\prime}-$ & 0.33 & 2.22 & $0.003^{d}$ & $0.0005^{d}$ & \\
\hline Dichlorophenol; 2,4- & 0.33 & 240 & NV & 2.65 & \\
\hline Dimethylphenol; 2,4- & 0.33 & 1,600 & 2.62 & 4.52 & \\
\hline Dimethylphthalate & 0.33 & 64,000 & 72.2 & 130 & \\
\hline $\begin{array}{l}\text { Di-nitro-2-methylphenol; } \\
4,6 \text { - }\end{array}$ & 0.33 & 8.00 & $0.0256^{d}$ & NV & \\
\hline Dinitrophenol; 2,4- & 0.825 & 160 & $0.125^{d}$ & $0.560^{d}$ & \\
\hline Dinitrotoluene; 2,4- & 0.33 & 160 & $0.189^{d}$ & $0.0005^{d}$ & \\
\hline
\end{tabular}


Table 4. Master 100-N Area Target Analytes, Analytical Methods, and Laboratory Detection Limițs. (7 Pages)

\begin{tabular}{|c|c|c|c|c|c|}
\hline \multirow[b]{2}{*}{ Target Analyte } & \multirow{2}{*}{$\begin{array}{c}\text { Practical } \\
\text { Quantitation }_{\text {Limits }^{a}} \\
\end{array}$} & \multicolumn{3}{|c|}{ Preliminary Cleanup Goals ${ }^{a, b}$} & \multirow{2}{*}{$\begin{array}{l}\text { Analytical } \\
\text { Methods }\end{array}$} \\
\hline & & $\begin{array}{c}\text { Direct } \\
\text { Exposure }\end{array}$ & $\begin{array}{c}\text { Groundwater } \\
\text { Protection }\end{array}$ & $\begin{array}{c}\text { River } \\
\text { Protection } \\
\end{array}$ & \\
\hline Dinitrotoluene; 2,6- & 0.33 & 80 & NV & NV & \multirow{17}{*}{$\begin{array}{l}\text { EPA } 8270 \\
\text { (SVOCs) }\end{array}$} \\
\hline Hexachlorobenzene & 0.33 & 0.625 & $0.015^{d}$ & $0.001^{d}$ & \\
\hline Hexachlorobutadiene & 0.33 & 12.8 & 0.605 & 0.475 & \\
\hline Hexachlorocyclopentadiene & 0.33 & 480 & NV & 960 & \\
\hline Hexachloroethane & 0.33 & 71.4 & 0.125 & 0.076 & \\
\hline Methylphenol; 2-(cresol; o-) & 0.33 & 4,000 & 5.14 & 5.15 & \\
\hline Nitroaniline; 2- & 0.33 & 240 & 0.121 & NV & \\
\hline Nitroaniline; 3- & 0.33 & 47.6 & 0.010 & NV & \\
\hline Nitroaniline; 4- & 0.33 & 47.6 & 0.010 & NV & \\
\hline Nitrobenzene & 0.33 & 160 & $0.102^{d}$ & $0.109^{d}$ & \\
\hline Nitrophenol; 2- & 0.66 & NV & NV & NV & \\
\hline Nitrophenol; 4- & 0.66 & 640 & 1.30 & 128 & \\
\hline $\begin{array}{l}\text { Nitroso-di-n-propylamine; } \\
\mathrm{N} \text { - }\end{array}$ & 0.33 & $0.143^{d}$ & 0.000056 & 0.0035 & \\
\hline Pentachlorophenol & 0.33 & 8.33 & $0.016^{d}$ & $0.00444^{d}$ & \\
\hline Trichlorophenol; 2,4,5- & 0.33 & 8,000 & 28.8 & NV & \\
\hline Trichlorophenol; 2,4,6- & 0.33 & 90.9 & $0.0462^{d}$ & $0.049^{d}$ & \\
\hline Nitrosodiphenylamine; $\mathrm{N}$ - & 0.33 & 204 & NV & NV & \\
\hline Acenaphthene & 0.1 & 4,800 & 97.9 & 65.5 & \multirow{13}{*}{$\begin{array}{l}\text { EPA-8310 } \\
\text { (PAHs) }\end{array}$} \\
\hline Acenaphthylene & 0.1 & 4,800 & 97.9 & 65.5 & \\
\hline Anthracene & 0.05 & 24,000 & 2.270 & 4,550 & \\
\hline Benzo(a)anthracene & 0.015 & 1.37 & 0.856 & 0.02400 & \\
\hline Benzo(a)pyrene & 0.015 & 0.137 & 2.33 & 0.0545 & \\
\hline Benzo(b)fluoranthene & 0.015 & 1.37 & 2.95 & 0.069 & \\
\hline Benzo(ghi)perylene & 0.03 & 2,400 & 25,700 & 3,540 & \\
\hline Benzo(k)fluoranthene & 0.015 & 1.37 & 21.5 & 0.069 & \\
\hline Chrysene & 0.1 & 13.7 & 9.56 & $0.0223^{d}$ & \\
\hline Dibenz(a,h)anthracene & 0.3 & 1.37 & 4.29 & 0.1 & \\
\hline Fluoranthene & 0.05 & 3,200 & 631 & 89 & \\
\hline Fluorene & 0.03 & 3,200 & 101 & 206 & \\
\hline Indeno(1,2,3-cd)pyrene & 0.03 & 1.37 & 8.33 & 0.195 & \\
\hline
\end{tabular}


Table 4. Master 100-N Area Target Analytes, Analytical Methods, and Laboratory Detection Limits. (7 Pages)

\begin{tabular}{|c|c|c|c|c|c|}
\hline \multirow[b]{2}{*}{ Target Analyte } & \multirow{2}{*}{$\begin{array}{c}\text { Practical } \\
\text { Quantitation } \\
\text { Limits }^{a}\end{array}$} & \multicolumn{3}{|c|}{ Preliminary Cleanup Goals ${ }^{a, b}$} & \multirow{2}{*}{$\begin{array}{l}\text { Analytical } \\
\text { Methods }\end{array}$} \\
\hline & & $\begin{array}{c}\text { Direct } \\
\text { Exposure }\end{array}$ & $\begin{array}{l}\text { Groundwater } \\
\text { Protection }\end{array}$ & $\begin{array}{c}\text { River } \\
\text { Protection }\end{array}$ & \\
\hline Naphthalene & 0.1 & 1,600 & 4.46 & 138 & \multirow{3}{*}{$\begin{array}{l}\text { EPA-8310 } \\
\text { (PAHs) }\end{array}$} \\
\hline Phenanthrene & 0.05 & 24,000 & 1,140 & 4,5500 & \\
\hline Pyrene & 0.05 & 2,400 & 655 & 1,3100 & \\
\hline Cyanide & 0.5 & 1,600 & 0.80 & 0.8 & $\begin{array}{l}\text { EPA } 9010 \text { or } \\
9012\end{array}$ \\
\hline Asbestos & $1 \%$ & NA & NA & NA & Microscopy \\
\hline TPH/diesel and motor oil & 5 & 2,000 & 2,000 & NV & NWTPH-D+ \\
\hline TPH/gasoline range & 5 & 30 & 30 & 30 & NWTPH-G \\
\hline
\end{tabular}

${ }^{a}$ Units are $\mathrm{mg} / \mathrm{kg}$ (nonradionuclides) and $\mathrm{pCi} / \mathrm{g}$ (radionuclides) unless otherwise noted. Cleanup levels are established in the most current CLARC table (updated February 12, 2009) calculated per Washington Administrative Code 173-340 (Ecology 2007) using input parameters stated in the CLARC table.

b Where cleanup levels are less than background, cleanup levels default to background as discussed in Section 2.1.2.1 of the 100 Area RDR/RAWP (DOE-RL-96-17, Rev 5).

c If strong gamma emissions interfere with analysis of Am-241, Am-241 can be analyzed using Cm/Am Alpha Emission Analysis method.

d Where cleanup levels are less than PQLs, cleanup levels default to PQLs as discussed in Section 2.1.2.1 of the 100 Area RDR/RAWP (DOE-RL-96-17, Rev 5).

Reference: Ecology, 2007, "Model Toxics Control Act Statute and Regulation," Publication No. 94-06, revised November 2007, Washington State Department of Ecology, Olympia, Washington.
$\mathrm{AEA}=$ alpha energy analysis
NWTPH = Northwest total petroleum hydrocarbons
CLARC $=$ Cleanup Levels and Risk Calculation
$\mathrm{PAH} \quad=$ polycyclic aromatic hydrocarbons
EPA = U.S. Environmental Protection Agency
PCB = polychlorinated biphenyl
$\mathrm{GC} \quad=$ gas chromatograph
GEA = gamma energy analysis
IC = ion chromatograph
PQL = practical quantitation limit
ICP = inductively coupled plasma
MS = mass spectroscopy
NA = not applicable
RDR/RAWP $=$ remedial design report/remedial action work plan
SVOA = semivolatile organic analysis
TBD = to be determined
$\mathrm{TPH}=$ total petroleum hydrocarbons
VOC = volatile organic compound

NV = No value. The generic RESidual RADioactivity modeling reported in the 100 Area RDR/RAWP predicts the contaminant will not reach groundwater within 1,000 years.

4. This step reconciles the master soil target analytes with the groundwater COPCs developed for the DU. Groundwater COPCs not found on the master soils list are further evaluated. The default action is to include all groundwater COPCs on the master soil target analyte list, unless there is a valid basis for their exclusion. Groundwater COPCs added to Table 4 are italicized.

5. The appropriate analytical methods for the master target analytes, taking into account action levels and detection limits, are presented in Table 5. 


\section{Step 2 - Location Specific Target Analyte Identification (per agreement with regulators,} Step 2 was not completed.)

1. Identify the contaminants of concern for the specific waste sites where characterization is proposed from the applicable interim action ROD (which reflects information from LFI and technical baseline reports). If the characterization location is not at a waste site, evaluate information from waste sites in the vicinity (where available). Include these analytes on the location specific target analyte list (Tables 5 through 8 ).

2. Identify the contaminants of concern for the specific waste site locations from the verification documentation (CVPS or RSVPS). If the characterization location is not at a waste site, evaluate information from waste sites in the vicinity (where available). Include these analytes on the location specific target analyte list (Tables 5 through 8).

3. Evaluate local groundwater monitoring well data (wells located within waste site "zones of influence"). Determine if groundwater COPCs have been analyzed for in these wells.

a. If the groundwater COPCs have been analyzed for but not detected, then these analytes will not be included on the location specific target analyte list.

b. If the groundwater COPCs have been analyzed for and have been detected, then these analytes are included on the location specific target analyte list.

c. If the groundwater COPCs have not been analyzed for, then an additional evaluation will be performed to determine if there is a data need. If there is a data need, these COPCs are included on the location specific target analyte list.

Table 5. Wells \#1 and \#R1 Target Analytes, Analytical Methods, and Contract-Required Detection Limits. (4 Pages)

\begin{tabular}{|c|c|c|c|c|c|}
\hline \multirow[b]{2}{*}{ Target Analyte } & \multirow{2}{*}{$\begin{array}{c}\text { Practical } \\
\text { Quantitation } \\
\text { Limits }^{a}\end{array}$} & \multicolumn{3}{|c|}{ Preliminary Cleanup Goals ${ }^{a, b}$} & \multirow{2}{*}{$\begin{array}{l}\text { Analytical } \\
\text { Methods }\end{array}$} \\
\hline & & $\begin{array}{c}\text { Direct } \\
\text { Exposure }\end{array}$ & $\begin{array}{c}\text { Groundwater } \\
\text { Protection }\end{array}$ & $\begin{array}{c}\text { River } \\
\text { Protection }\end{array}$ & \\
\hline \multicolumn{6}{|c|}{ Radionuclides } \\
\hline Cesium-137 & 0.1 & 6.2 & 1,465 & 2,930 & $\begin{array}{l}\text { EPA } 8310 \\
\text { (PAHs) }\end{array}$ \\
\hline Cobalt -60 & 0.05 & 1.4 & 13,900 & 27,800 & $\begin{array}{l}\text { EPA } 8310 \\
\text { (PAHs) }\end{array}$ \\
\hline Strontium-90 & 1 & 4.5 & 27.6 & 55.2 & $\begin{array}{l}\text { Gas flow } \\
\text { proportional } \\
\text { counting }\end{array}$ \\
\hline lodine-129 & 2 & $2^{b}$ & $2^{b}$ & $2^{b}$ & Low energy GEA \\
\hline Plutonium-239/240 & 1 & 33.9 & NV & NV & Isotopic PU AEA \\
\hline Carbon-14 & 2 & 8.7 & NV & NV & $\begin{array}{l}\text { Liquid } \\
\text { scintillation } \\
\text { counting }\end{array}$ \\
\hline Technetium-99 & 0.25 & 5.7 & 0.46 & 0.46 & $\begin{array}{l}\text { Liquid } \\
\text { scintillation } \\
\text { counting }\end{array}$ \\
\hline
\end{tabular}


Table 5. Wells \#1 and \#R1 Target Analytes, Analytical Methods, and Contract-Required Detection Limits. (4 Pages)

\begin{tabular}{|c|c|c|c|c|c|}
\hline \multirow{2}{*}{ Target Analyte } & \multirow{2}{*}{$\begin{array}{c}\text { Practical } \\
\text { Quantitation } \\
\text { Limits }^{a}\end{array}$} & \multicolumn{3}{|c|}{ Preliminary Cleanup Goals ${ }^{a, b}$} & \multirow{2}{*}{$\begin{array}{c}\text { Analytical } \\
\text { Methods }\end{array}$} \\
\hline & & $\begin{array}{c}\text { Direct } \\
\text { Exposure }\end{array}$ & $\begin{array}{l}\text { Groundwater } \\
\text { Protection }\end{array}$ & $\begin{array}{c}\text { River } \\
\text { Protection }\end{array}$ & \\
\hline Tritium & 10 & 510 & 12.6 & 25.2 & $\begin{array}{l}\text { Liquid } \\
\text { scintillation } \\
\text { counting } \\
\end{array}$ \\
\hline \multicolumn{6}{|c|}{ Nonradionuclides } \\
\hline Fluoride & 5 & 4,800 & 2,800 & 2,890 & $\begin{array}{l}\text { EPA } 300.0 \\
\text { (anions by IC) }\end{array}$ \\
\hline Nitrate (as nitrogen) & 2.5 & 128,000 & 40 & 40 & $\begin{array}{l}\text { EPA } 300.0 \\
\text { (anions by IC) }\end{array}$ \\
\hline Nitrite (as nitrogen) & 2.5 & 8,000 & 40 & 4 & $\begin{array}{l}\text { EPA } 300.0 \\
\text { (anions by IC) }\end{array}$ \\
\hline Sulfate & 5 & NV & 1,030 & 1,030 & $\begin{array}{l}\text { EPA } 300.0 \\
\text { (anions by IC) }\end{array}$ \\
\hline Aluminum & 5 & 80,000 & 480,000 & 480,000 & $\begin{array}{l}\text { EPA } 6010 \text { or } \\
200.8 \text { (ICP or } \\
\text { ICP/MS) }\end{array}$ \\
\hline Antimony & 6 & 32 & 5.4 & 12.7 & $\begin{array}{l}\text { EPA } 6010 \text { or } \\
200.8 \text { (ICP or } \\
\text { ICP/MS) }\end{array}$ \\
\hline Arsenic & 10 & $\mathrm{TBD}^{\mathrm{b}}$ & $\mathrm{TBD}^{\mathrm{b}}$ & $\mathrm{TBD}^{\mathrm{b}}$ & $\begin{array}{l}\text { EPA } 6010 \text { or } \\
200.8 \text { (ICP or } \\
\text { ICP/MS) }\end{array}$ \\
\hline Barium & 2 & 16,000 & 1,650 & 1,600 & $\begin{array}{l}\text { EPA } 6010 \text { or } \\
200.8 \text { (ICP or } \\
\text { ICP/MS) }\end{array}$ \\
\hline Beryllium & 0.5 & 160 & 63.2 & 63 & $\begin{array}{l}\text { EPA } 6010 \text { or } \\
200.8 \text { (ICP or } \\
\text { ICP/MS) }\end{array}$ \\
\hline Boron & 2 & 16,000 & 210 & NV & $\begin{array}{l}\text { EPA } 6010 \text { or } \\
200.8 \text { (ICP or } \\
\text { ICP/MS) }\end{array}$ \\
\hline Chromium (total) & 1 & 120,000 & 2,000 & 1,300 & $\begin{array}{l}\text { EPA } 6010 \text { or } \\
200.8 \text { (ICP or } \\
\text { ICP/MS) }\end{array}$ \\
\hline Cobalt & 2 & 24 & 15.7 & NV & $\begin{array}{l}\text { EPA } 6010 \text { or } \\
200.8 \text { (ICP or } \\
\text { ICP/MS) }\end{array}$ \\
\hline Copper & 1 & 3,200 & 284 & 575 & $\begin{array}{l}\text { EPA } 6010 \text { or } \\
200.8 \text { (ICP or } \\
\text { ICP/MS) }\end{array}$ \\
\hline Lead & 5 & 250 & 3,000 & 420 & $\begin{array}{l}\text { EPA } 6010 \text { or } \\
200.8 \text { (ICP or } \\
\text { ICP/MS) }\end{array}$ \\
\hline Lithium & 2.5 & 160 & 192 & NV & $\begin{array}{l}\text { EPA } 6010 \text { or } \\
200.8 \text { (ICP or } \\
\text { ICP/MS) }\end{array}$ \\
\hline Manganese & 5 & 3,760 & $512^{b}$ & $256^{\mathrm{b}}$ & $\begin{array}{l}\text { EPA } 6010 \text { or } \\
200.8 \text { (ICP or } \\
\text { ICP/MS) }\end{array}$ \\
\hline
\end{tabular}


Table 5. Wells \#1 and \#R1 Target Analytes, Analytical Methods, and Contract-Required Detection Limits. (4 Pages)

\begin{tabular}{|c|c|c|c|c|c|}
\hline \multirow[b]{2}{*}{ Target Analyte } & \multirow{2}{*}{$\begin{array}{l}\text { Practical } \\
\text { Quantitation } \\
\text { Limits }^{a}\end{array}$} & \multicolumn{3}{|c|}{ Preliminary Cleanup Goals ${ }^{a, b}$} & \multirow{2}{*}{$\begin{array}{l}\text { Analytical } \\
\text { Methods }\end{array}$} \\
\hline & & $\begin{array}{c}\text { Direct } \\
\text { Exposure }\end{array}$ & $\begin{array}{l}\text { Groundwater } \\
\text { Protection }\end{array}$ & $\begin{array}{c}\text { River } \\
\text { Protection }\end{array}$ & \\
\hline Nickel & 4 & 1,600 & 130 & 179 & $\begin{array}{l}\text { EPA } 6010 \text { or } \\
200.8 \text { (ICP or } \\
\text { ICP/MS) }\end{array}$ \\
\hline Selenium & 10 & 400 & $5.2^{\mathrm{c}}$ & $0.52^{c}$ & $\begin{array}{l}\text { EPA } 6010 \text { or } \\
200.8 \text { (ICP or } \\
\text { ICP/MS) }\end{array}$ \\
\hline Silver & 1 & 400 & 13.6 & 0.442 & $\begin{array}{l}\text { EPA } 6010 \text { or } \\
200.8 \text { (ICP or } \\
\text { ICP/MS) }\end{array}$ \\
\hline Vanadium & 2.5 & 560 & 2,240 & NV & $\begin{array}{l}\text { EPA } 6010 \text { or } \\
200.8 \text { (ICP or } \\
\text { ICP/MS) }\end{array}$ \\
\hline Zinc & 1 & 24,000 & 5,970 & 226 & $\begin{array}{l}\text { EPA } 6010 \text { or } \\
200.8 \text { (ICP or } \\
\text { ICP/MS) }\end{array}$ \\
\hline Chromium (hexavalent) & 0.5 & TBD & TBD & TBD & $\begin{array}{l}\text { EPA } 7196 \\
\text { (hexavalent } \\
\text { chromium) }\end{array}$ \\
\hline Mercury & 0.2 & 24 & 2.09 & $0.33^{b}$ & $\begin{array}{l}\text { EPA } 7471(\mathrm{Hg} \\
\text { cold vapor) or } \\
200.8 \text { (ICP/MS) }\end{array}$ \\
\hline Chloroform & 0.005 & 164 & 0.038 & 0.0304 & $\begin{array}{l}\text { EPA } 8260 \\
\text { (VOCs) }\end{array}$ \\
\hline Tetrachloroethene & 0.005 & 1.85 & 0.008 & 0.004 & $\begin{array}{l}\text { EPA } 8260 \\
\text { (VOCs) }\end{array}$ \\
\hline Acenaphthene & 0.1 & 4,800 & 97.9 & 65.5 & $\begin{array}{l}\text { EPA } 8310 \\
\text { (PAHs) }\end{array}$ \\
\hline Acenaphthylene & 0.1 & 4,800 & 97.9 & 65.5 & $\begin{array}{l}\text { EPA } 8310 \\
\text { (PAHs) }\end{array}$ \\
\hline Anthracene & 0.05 & 24,000 & 2.270 & 4,550 & $\begin{array}{l}\text { EPA 8310 } \\
\text { (PAHs) }\end{array}$ \\
\hline Benzo(a)anthracene & 0.015 & 1.37 & 0.856 & 0.02400 & $\begin{array}{l}\text { EPA } 8310 \\
\text { (PAHs) }\end{array}$ \\
\hline Benzo(a)pyrene & 0.015 & 0.137 & 2.33 & 0.0545 & $\begin{array}{l}\text { EPA } 8310 \\
\text { (PAHs) }\end{array}$ \\
\hline Benzo(b)fluoranthene & 0.015 & 1.37 & 2.95 & 0.069 & $\begin{array}{l}\text { EPA } 8310 \\
\text { (PAHs) }\end{array}$ \\
\hline Benzo(ghi)perylene & 0.03 & 2,400 & 25,700 & 3,540 & $\begin{array}{l}\text { EPA } 8310 \\
\text { (PAHs) }\end{array}$ \\
\hline Benzo(k)fluoranthene & 0.015 & 1.37 & 21.5 & 0.069 & $\begin{array}{l}\text { EPA } 8310 \\
\text { (PAHs) }\end{array}$ \\
\hline Chrysene & 0.1 & 13.7 & 9.56 & $0.0223^{c}$ & $\begin{array}{l}\text { EPA } 8310 \\
\text { (PAHs) }\end{array}$ \\
\hline Dibenz(a,h)anthracene & 0.3 & 1.37 & 4.29 & 0.1 & $\begin{array}{l}\text { EPA 8310 } \\
\text { (PAHs) }\end{array}$ \\
\hline Fluoranthene & 0.05 & 3,200 & 631 & 89 & $\begin{array}{l}\text { EPA } 8310 \\
\text { (PAHs) }\end{array}$ \\
\hline
\end{tabular}


Table 5. Wells \#1 and \#R1 Target Analytes, Analytical Methods, and Contract-Required Detection Limits. (4 Pages)

\begin{tabular}{|c|c|c|c|c|c|}
\hline \multirow[b]{2}{*}{ Target Analyte } & \multirow{2}{*}{$\begin{array}{c}\text { Practical } \\
\text { Quantitation } \\
\text { Limits }^{\text {a }}\end{array}$} & \multicolumn{3}{|c|}{ Preliminary Cleanup Goals ${ }^{a, b}$} & \multirow{2}{*}{$\begin{array}{l}\text { Analytical } \\
\text { Methods }\end{array}$} \\
\hline & & $\begin{array}{c}\text { Direct } \\
\text { Exposure }\end{array}$ & $\begin{array}{l}\text { Groundwater } \\
\text { Protection }\end{array}$ & $\begin{array}{c}\text { River } \\
\text { Protection }\end{array}$ & \\
\hline Fluorene & 0.03 & 3,200 & 101 & 206 & $\begin{array}{l}\text { EPA } 8310 \\
\text { (PAHs) }\end{array}$ \\
\hline Indeno(1,2,3-cd)pyrene & 0.03 & 1.37 & 8.33 & 0.195 & $\begin{array}{l}\text { EPA } 8310 \\
\text { (PAHs) }\end{array}$ \\
\hline Naphthalene & 0.1 & 1,600 & 4.46 & 138 & $\begin{array}{l}\text { EPA } 8310 \\
\text { (PAHs) }\end{array}$ \\
\hline Phenanthrene & 0.05 & 24,000 & 1,140 & 4,5500 & $\begin{array}{l}\text { EPA } 8310 \\
\text { (PAHs) }\end{array}$ \\
\hline Pyrene & 0.05 & 2,400 & 655 & 1,3100 & $\begin{array}{l}\text { EPA } 8310 \\
\text { (PAHs) }\end{array}$ \\
\hline Cyanide & 0.5 & 1,600 & 0.80 & 0.8 & $\begin{array}{l}\text { EPA } 9010 \text { or } \\
9012\end{array}$ \\
\hline TPH/diesel and motor oil & 5 & 2,000 & 2,000 & NV & NWTPH-D+ \\
\hline
\end{tabular}

${ }^{a}$ Units are $\mathrm{mg} / \mathrm{kg}$ (nonradionuclides) and pCi/g (radionuclides) unless otherwise noted. Cleanup levels are established in the most current CLARC table (updated February 12, 2009) calculated per Washington Administrative Code 173-340 (Ecology 2007) using input parameters stated in the CLARC table.

b Where cleanup levels are less than background, cleanup levels default to background as discussed in Section 2.1.2.1 of the 100 Area RDR/RAWP (DOE-RL-96-17, Rev 5).

${ }^{\circ}$ Where cleanup levels are less than PQLs, cleanup levels default to PQLs as discussed in Section 2.1.2.1 of the 100 Area RDR/RAWP (DOE-RL-96-17, Rev 5).

Reference: Ecology, 2007, "Model Toxics Control Act Statute and Regulation," Publication No. 94-06, revised November 2007, Washington State Department of Ecology, Olympia, Washington.

AEA = alpha energy analysis

$C L A R C=$ Cleanup Levels and Risk Calculation

EPA = U.S. Environmental Protection Agency

GEA = gamma energy analysis

IC = ion chromatograph

ICP = inductively coupled plasma

MS = mass spectroscopy

NV = No value. The generic RESidual RADioactivity modeling reported in the 100 Area RDR/RAWP predicts the contaminant will not reach groundwater within 1,000 years.
NWTPH = Northwest total petroleum hydrocarbons

$\mathrm{PAH} \quad=$ polycyclic aromatic hydrocarbons

PQL $\quad=$ practical quantitation limit

RDR/RAWP = remedial design report/remedial action work plan

$\mathrm{TPH} \quad=$ total petroleum hydrocarbons

TBD = to be determined

VOC $\quad=$ volatile organic compound

Table 6. Well \#2 Target Analytes, Analytical Methods, and Contract-Required Detection Limits. (4 Pages)

\begin{tabular}{|l|c|c|c|c|l|}
\hline \multirow{2}{*}{ Target Analyte } & \multirow{2}{*}{$\begin{array}{c}\text { Practical } \\
\text { Quantitation } \\
\text { Limits }^{\mathrm{a}}\end{array}$} & \multicolumn{5}{|c|}{\begin{tabular}{c} 
Preliminary Cleanup Goals \\
\cline { 3 - 6 }
\end{tabular}} & $\begin{array}{c}\text { Direct } \\
\text { Exposure }\end{array}$ & $\begin{array}{c}\text { Groundwater } \\
\text { Protection }\end{array}$ & $\begin{array}{c}\text { River } \\
\text { Analytical } \\
\text { Protection }\end{array}$ & \\
\hline Madionuclides \\
\hline Cesium-137 & 0.1 & 6.2 & 1,465 & 2,930 & GEA \\
\hline Cobalt-60 & 0.05 & 1.4 & 13,900 & 27,800 & GEA \\
\hline Strontium-90 & 1 & 4.5 & 27.6 & 55.2 & $\begin{array}{l}\text { Gas flow } \\
\text { proportional } \\
\text { counting }\end{array}$ \\
\hline lodine-129 & 2 & $2^{b}$ & $2^{b}$ & $2^{b}$ & $\begin{array}{l}\text { Low energy } \\
\text { GEA }\end{array}$ \\
\hline
\end{tabular}


Table 6. Well \#2 Target Analytes, Analytical Methods, and Contract-Required Detection Limits. (4 Pages)

\begin{tabular}{|c|c|c|c|c|c|}
\hline \multirow{2}{*}{ Target Analyte } & \multirow{2}{*}{$\begin{array}{c}\text { Practical } \\
\text { Quantitation } \\
\text { Limits }^{a}\end{array}$} & \multicolumn{3}{|c|}{ Preliminary Cleanup Goals ${ }^{a, b}$} & \multirow{2}{*}{$\begin{array}{l}\text { Analytical } \\
\text { Methods }\end{array}$} \\
\hline & & $\begin{array}{c}\text { Direct } \\
\text { Exposure }\end{array}$ & $\begin{array}{l}\text { Groundwater } \\
\text { Protection }\end{array}$ & $\begin{array}{c}\text { River } \\
\text { Protection }\end{array}$ & \\
\hline Plutonium-239/240 & 1 & 33.9 & NV & NV & $\begin{array}{l}\text { Isotopic } \mathrm{Pu} \\
\mathrm{AEA}\end{array}$ \\
\hline Carbon-14 & 2 & 8.7 & NV & NV & $\begin{array}{l}\text { Liquid } \\
\text { scintillation } \\
\text { counting }\end{array}$ \\
\hline Technetium-99 & 0.25 & 5.7 & 0.46 & 0.46 & $\begin{array}{l}\text { Liquid } \\
\text { scintillation } \\
\text { counting }\end{array}$ \\
\hline Tritium & 10 & 510 & 12.6 & 25.2 & $\begin{array}{l}\text { Liquid } \\
\text { scintillation } \\
\text { counting }\end{array}$ \\
\hline \multicolumn{6}{|c|}{ Nonradionuclides } \\
\hline Fluoride & 5 & 4,800 & 2,800 & 2,890 & $\begin{array}{l}\text { EPA } 300.0 \\
\text { (anions by IC) }\end{array}$ \\
\hline Nitrate (as nitrogen) & 2.5 & 128,000 & 40 & 40 & $\begin{array}{l}\text { EPA } 300.0 \\
\text { (anions by IC) }\end{array}$ \\
\hline Nitrite (as nitrogen) & 2.5 & 8,000 & 40 & 4 & $\begin{array}{l}\text { EPA } 300.0 \\
\text { (anions by IC) }\end{array}$ \\
\hline Sulfate & 5 & NV & 1,030 & 1,030 & $\begin{array}{l}\text { EPA } 300.0 \\
\text { (anions by IC) }\end{array}$ \\
\hline Aluminum & 5 & 80,000 & 480,000 & 480,000 & $\begin{array}{l}\text { EPA } 6010 \text { or } \\
200.8 \text { (ICP or } \\
\text { ICP/MS) }\end{array}$ \\
\hline Antimony & 6 & 32 & 5.4 & 12.7 & $\begin{array}{l}\text { EPA } 6010 \text { or } \\
200.8 \text { (ICP or } \\
\text { ICP/MS) }\end{array}$ \\
\hline Arsenic & 10 & $\mathrm{TBD}^{\mathrm{b}}$ & $\mathrm{TBD}^{\mathrm{b}}$ & $\mathrm{TBD}^{\mathrm{b}}$ & $\begin{array}{l}\text { EPA } 6010 \text { or } \\
200.8 \text { (ICP or } \\
\text { ICP/MS) }\end{array}$ \\
\hline Barium & 2 & 16,000 & 1,650 & 1,600 & $\begin{array}{l}\text { EPA } 6010 \text { or } \\
200.8 \text { (ICP or } \\
\text { ICP/MS) }\end{array}$ \\
\hline Beryllium & 0.5 & 160 & 63.2 & 63 & $\begin{array}{l}\text { EPA } 6010 \text { or } \\
200.8 \text { (ICP or } \\
\text { ICP/MS) }\end{array}$ \\
\hline Boron & 2 & 16,000 & 210 & NV & $\begin{array}{l}\text { EPA } 6010 \text { or } \\
200.8 \text { (ICP or } \\
\text { ICP/MS) }\end{array}$ \\
\hline Chromium (total) & 1 & 120,000 & 2,000 & 1,300 & $\begin{array}{l}\text { EPA } 6010 \text { or } \\
200.8 \text { (ICP or } \\
\text { ICP/MS) }\end{array}$ \\
\hline Cobalt & 2 & 24 & 15.7 & NV & $\begin{array}{l}\text { EPA } 6010 \text { or } \\
200.8 \text { (ICP or } \\
\text { ICP/MS) }\end{array}$ \\
\hline Copper & 1 & 3,200 & 284 & 575 & $\begin{array}{l}\text { EPA } 6010 \text { or } \\
200.8 \text { (ICP or } \\
\text { ICP/MS) }\end{array}$ \\
\hline
\end{tabular}


Table 6. Well \#2 Target Analytes, Analytical Methods, and Contract-Required Detection Limits. (4 Pages)

\begin{tabular}{|c|c|c|c|c|c|}
\hline \multirow[b]{2}{*}{ Target Analyte } & \multirow{2}{*}{$\begin{array}{c}\text { Practical } \\
\text { Quantitation }_{\text {Limits }^{a}}\end{array}$} & \multicolumn{3}{|c|}{ Preliminary Cleanup Goals ${ }^{a, b}$} & \multirow{2}{*}{$\begin{array}{l}\text { Analytical } \\
\text { Methods }\end{array}$} \\
\hline & & $\begin{array}{c}\text { Direct } \\
\text { Exposure }\end{array}$ & $\begin{array}{l}\text { Groundwater } \\
\text { Protection }\end{array}$ & $\begin{array}{c}\text { River } \\
\text { Protection }\end{array}$ & \\
\hline Lead & 5 & 250 & 3,000 & 420 & $\begin{array}{l}\text { EPA } 6010 \text { or } \\
200.8 \text { (ICP or } \\
\text { ICP/MS) }\end{array}$ \\
\hline Lithium & 2.5 & 160 & 192 & NV & $\begin{array}{l}\text { EPA } 6010 \text { or } \\
200.8 \text { (ICP or } \\
\text { ICP/MS) }\end{array}$ \\
\hline Manganese & 5 & 3,760 & $512^{b}$ & $256^{b}$ & $\begin{array}{l}\text { EPA } 6010 \text { or } \\
200.8 \text { (ICP or } \\
\text { ICP/MS) }\end{array}$ \\
\hline Nickel & 4 & 1,600 & 130 & 179 & $\begin{array}{l}\text { EPA } 6010 \text { or } \\
200.8 \text { (ICP or } \\
\text { ICP/MS) }\end{array}$ \\
\hline Selenium & 10 & 400 & $5.2^{\mathrm{c}}$ & $0.52^{\mathrm{c}}$ & $\begin{array}{l}\text { EPA } 6010 \text { or } \\
200.8 \text { (ICP or } \\
\text { ICP/MS) }\end{array}$ \\
\hline Silver & 1 & 400 & 13.6 & 0.442 & $\begin{array}{l}\text { EPA } 6010 \text { or } \\
200.8 \text { (ICP or } \\
\text { ICP/MS) }\end{array}$ \\
\hline Vanadium & 2.5 & 560 & 2,240 & NV & $\begin{array}{l}\text { EPA } 6010 \text { or } \\
200.8 \text { (ICP or } \\
\text { ICP/MS) }\end{array}$ \\
\hline Zinc & 1 & 24,000 & 5,970 & 226 & $\begin{array}{l}\text { EPA } 6010 \text { or } \\
200.8 \text { (ICP or } \\
\text { ICP/MS) }\end{array}$ \\
\hline Chromium (hexavalent) & 0.5 & TBD & TBD & TBD & $\begin{array}{l}\text { EPA } 7196 \\
\text { (hexavalent } \\
\text { chromium) }\end{array}$ \\
\hline Mercury & 0.2 & 24 & 2.09 & $0.33^{b}$ & $\begin{array}{l}\text { EPA } 7471 \\
\text { (Hg cold } \\
\text { vapor) }\end{array}$ \\
\hline Benzene & 0.005 & 18.2 & 0.004 & 0.007 & $\begin{array}{l}\text { EPA } 8260 \\
\text { (VOCs) }\end{array}$ \\
\hline Ethylbenzene & 0.005 & 8,000 & 6.05 & 26.8 & $\begin{array}{l}\text { EPA } 8260 \\
\text { (VOCs) }\end{array}$ \\
\hline Toluene & 0.005 & 6,400 & 4.65 & 49.5 & $\begin{array}{l}\text { EPA } 8260 \\
\text { (VOCs) }\end{array}$ \\
\hline Xylenes (total) & 0.01 & 16,000 & 3.09 & 5.15 & $\begin{array}{l}\text { EPA } 8260 \\
\text { (VOCs) }\end{array}$ \\
\hline Acenaphthene & 0.1 & 4,800 & 97.9 & 65.5 & $\begin{array}{l}\text { EPA-8310 } \\
\text { (PAHS) }\end{array}$ \\
\hline Acenaphthylene & 0.1 & 4,800 & 97.9 & 65.5 & $\begin{array}{l}\text { EPA-8310 } \\
\text { (PAHs) }\end{array}$ \\
\hline Anthracene & 0.05 & 24,000 & 2.270 & 4,550 & $\begin{array}{l}\text { EPA-8310 } \\
\text { (PAHs) }\end{array}$ \\
\hline Benzo(a)anthracene & 0.015 & 1.37 & 0.856 & 0.02400 & $\begin{array}{l}\text { EPA-8310 } \\
\text { (PAHs) }\end{array}$ \\
\hline Benzo(a)pyrene & 0.015 & 0.137 & 2.33 & 0.0545 & $\begin{array}{l}\text { EPA-8310 } \\
\text { (PAHs) }\end{array}$ \\
\hline
\end{tabular}


Table 6. Well \#2 Target Analytes, Analytical Methods, and Contract-Required Detection Limits. (4 Pages)

\begin{tabular}{|c|c|c|c|c|c|}
\hline \multirow[b]{2}{*}{ Target Analyte } & \multirow{2}{*}{$\begin{array}{c}\text { Practical } \\
\text { Quantitation } \\
\text { Limits }^{a}\end{array}$} & \multicolumn{3}{|c|}{ Preliminary Cleanup Goals ${ }^{a, b}$} & \multirow{2}{*}{$\begin{array}{l}\text { Analytical } \\
\text { Methods }\end{array}$} \\
\hline & & $\begin{array}{c}\text { Direct } \\
\text { Exposure }\end{array}$ & $\begin{array}{l}\text { Groundwater } \\
\text { Protection }\end{array}$ & $\begin{array}{c}\text { River } \\
\text { Protection }\end{array}$ & \\
\hline Benzo(b)fluoranthene & 0.015 & 1.37 & 2.95 & 0.069 & $\begin{array}{l}\text { EPA-8310 } \\
\text { (PAHs) }\end{array}$ \\
\hline Benzo(ghi)perylene & 0.03 & 2,400 & 25,700 & 3,540 & $\begin{array}{l}\text { EPA-8310 } \\
\text { (PAHs) }\end{array}$ \\
\hline Benzo(k)fluoranthene & 0.015 & 1.37 & 21.5 & 0.069 & $\begin{array}{l}\text { EPA-8310 } \\
\text { (PAHs) }\end{array}$ \\
\hline Chrysene & 0.1 & 13.7 & 9.56 & $0.0223^{c}$ & $\begin{array}{l}\text { EPA-8310 } \\
\text { (PAHs) }\end{array}$ \\
\hline Dibenz(a,h)anthracene & 0.3 & 1.37 & 4.29 & 0.1 & $\begin{array}{l}\text { EPA-8310 } \\
\text { (PAHs) }\end{array}$ \\
\hline Fluoranthene & 0.05 & 3,200 & 631 & 89 & $\begin{array}{l}\text { EPA-8310 } \\
\text { (PAHs) }\end{array}$ \\
\hline Fluorene & 0.03 & 3,200 & 101 & 206 & $\begin{array}{l}\text { EPA-8310 } \\
\text { (PAHs) }\end{array}$ \\
\hline Indeno(1,2,3-cd) pyrene & 0.03 & 1.37 & 8.33 & 0.195 & $\begin{array}{l}\text { EPA-8310 } \\
\text { (PAHs) }\end{array}$ \\
\hline Naphthalene & 0.1 & 1,600 & 4.46 & 138 & $\begin{array}{l}\text { EPA-8310 } \\
\text { (PAHs) }\end{array}$ \\
\hline Phenanthrene & 0.05 & 24,000 & 1,140 & 4,5500 & $\begin{array}{l}\text { EPA-8310 } \\
\text { (PAHs) }\end{array}$ \\
\hline Pyrene & 0.05 & 2,400 & 655 & 1,3100 & $\begin{array}{l}\text { EPA-8310 } \\
\text { (PAHs) }\end{array}$ \\
\hline $\begin{array}{l}\mathrm{TPH} / \text { diesel and motor } \\
\text { oil }\end{array}$ & 5 & 2,000 & 2,000 & NV & NWTPH-D+ \\
\hline TPH/gasoline range & 5 & 30 & 30 & 30 & NWTPH-G \\
\hline
\end{tabular}

${ }^{a}$ Units are $\mathrm{mg} / \mathrm{kg}$ (nonradionuclides) and $\mathrm{pCi} / \mathrm{g}$ (radionuclides) unless otherwise noted. Cleanup levels are established in the most current CLARC table (updated February 12, 2009) calculated per Washington Administrative Code 173-340 (Ecology 2007) using input parameters stated in the CLARC table.

b Where cleanup levels are less than background, cleanup levels default to background as discussed in Section 2.1.2.1 of the 100 Area RDR/RAWP (DOE-RL-96-17, Rev 5).

${ }^{c}$ Where cleanup levels are less than PQLs, cleanup levels default to PQLs as discussed in Section 2.1.2.1 of the 100 Area RDR/RAWP (DOE-RL-96-17, Rev 5).

Reference: Washington State Department of Ecology, 2007, "Model Toxics Control Act Statute and Regulation," Publication No. 94-06, revised November 2007, Washington State Department of Ecology, Olympia, Washington.

AEA = alpha energy analysis

$\mathrm{CLARC}=$ Cleanup Levels and Risk Calculation

EPA $=$ U.S. Environmental Protection Agency

GEA = gamma energy analysis

IC = ion chromatograph

ICP = inductively coupled plasma

MS = mass spectroscopy

NWTPH = northwest total petroleum hydrocarbons

$\mathrm{PAH} \quad=$ polycyclic aromatic hydrocarbons

$P Q L \quad=$ practical quantitation limit

RDR/RAWP = remedial design report/remedial action work plan

TBD $\quad=$ to be determined

$\mathrm{TPH}=$ total petroleum hydrocarbons

VOC = volatile organic compound

NV = No value. The generic RESidual RADioactivity modeling reported in the 100 Area RDR/RAWP predicts the contaminant will not reach groundwater within 1,000 years. 
Table 7. Wells \#6 and \#R2 Target Analytes, Analytical Methods, and Contract-Required Detection Limits. (2 Pages)

\begin{tabular}{|c|c|c|c|c|c|}
\hline \multirow[b]{2}{*}{ Target Analyte } & \multirow{2}{*}{$\begin{array}{c}\text { Practical } \\
\begin{array}{c}\text { Quantitation } \\
\text { Limits }^{\mathrm{a}}\end{array}\end{array}$} & \multicolumn{3}{|c|}{ Preliminary Cleanup Goals ${ }^{a, b}$} & \multirow{2}{*}{$\begin{array}{l}\text { Analytical } \\
\text { Methods }\end{array}$} \\
\hline & & $\begin{array}{c}\text { Direct } \\
\text { Exposure }\end{array}$ & $\begin{array}{l}\text { Groundwater } \\
\text { Protection }\end{array}$ & $\begin{array}{c}\text { River } \\
\text { Protection }\end{array}$ & \\
\hline \multicolumn{6}{|c|}{ Radionuclides } \\
\hline Cesium-137 & 0.1 & 6.2 & 1,465 & 2,930 & GEA \\
\hline Cobalt -60 & 0.05 & 1.4 & 13,900 & 27,800 & GEA \\
\hline Europium-152 & 0.1 & 3.3 & NV & NV & GEA \\
\hline Europium-154 & 0.1 & 3.0 & NV & NV & GEA \\
\hline Strontium-90 & 1 & 4.5 & 27.6 & 55.2 & $\begin{array}{l}\text { Gas flow } \\
\text { proportional } \\
\text { counting }\end{array}$ \\
\hline Tritium & 10 & 510 & 12.6 & 25.2 & $\begin{array}{l}\text { Liquid } \\
\text { scintillation } \\
\text { counting } \\
\end{array}$ \\
\hline \multicolumn{6}{|c|}{ Nonradionuclides } \\
\hline Antimony & 6 & 32 & 5.4 & 12.7 & $\begin{array}{l}\text { EPA } 6010 \text { or } \\
200.8 \text { (ICP or } \\
\text { ICP/MS) }\end{array}$ \\
\hline Arsenic & 10 & $\mathrm{TBD}^{\mathrm{b}}$ & $\mathrm{TBD}^{\mathrm{b}}$ & $\mathrm{TBD}^{\mathrm{b}}$ & $\begin{array}{l}\text { EPA } 6010 \text { or } \\
200.8 \text { (ICP or } \\
\text { ICP/MS) }\end{array}$ \\
\hline Beryllium & 0.5 & 160 & 63.2 & 63 & $\begin{array}{l}\text { EPA } 6010 \text { or } \\
200.8 \text { (ICP or } \\
\text { ICP/MS) }\end{array}$ \\
\hline Cadmium & 0.5 & 80 & 0.69 & $0.125^{\mathrm{c}}$ & $\begin{array}{l}\text { EPA } 6010 \text { or } \\
200.8 \text { (ICP or } \\
\text { ICP/MS) }\end{array}$ \\
\hline Chromium (total) & 1 & 120,000 & 2,000 & 1,300 & $\begin{array}{l}\text { EPA } 6010 \text { or } \\
200.8 \text { (ICP or } \\
\text { ICP/MS) }\end{array}$ \\
\hline Cobalt & 2 & 24 & 15.7 & NV & $\begin{array}{l}\text { EPA } 6010 \text { or } \\
200.8 \text { (ICP or } \\
\text { ICP/MS) }\end{array}$ \\
\hline Copper & 1 & 3,200 & 284 & 575 & $\begin{array}{l}\text { EPA } 6010 \text { or } \\
200.8 \text { (ICP or } \\
\text { ICP/MS) }\end{array}$ \\
\hline Lead & 5 & 250 & 3,000 & 420 & $\begin{array}{l}\text { EPA } 6010 \text { or } \\
200.8 \text { (ICP or } \\
\text { ICP/MS) }\end{array}$ \\
\hline Manganese & 5 & 3,760 & $512^{b}$ & $256^{\mathrm{b}}$ & $\begin{array}{l}\text { EPA } 6010 \text { or } \\
200.8 \text { (ICP or } \\
\text { ICP/MS) }\end{array}$ \\
\hline Nickel & 4 & 1,600 & 130 & 179 & $\begin{array}{l}\text { EPA } 6010 \text { or } \\
200.8 \text { (ICP or } \\
\text { ICP/MS) }\end{array}$ \\
\hline
\end{tabular}


Table 7. Wells \#6 and \#R2 Target Analytes, Analytical Methods, and Contract-Required Detection Limits. (2 Pages)

\begin{tabular}{|c|c|c|c|c|c|}
\hline \multirow[b]{2}{*}{ Target Analyte } & \multirow{2}{*}{$\begin{array}{c}\text { Practical } \\
\begin{array}{c}\text { Quantitation } \\
\text { Limits }^{a}\end{array}\end{array}$} & \multicolumn{3}{|c|}{ Preliminary Cleanup Goals ${ }^{a, b}$} & \multirow{2}{*}{$\begin{array}{l}\text { Analytical } \\
\text { Methods }\end{array}$} \\
\hline & & $\begin{array}{c}\text { Direct } \\
\text { Exposure }\end{array}$ & $\begin{array}{c}\text { Groundwater } \\
\text { Protection }\end{array}$ & $\begin{array}{c}\text { River } \\
\text { Protection }\end{array}$ & \\
\hline Selenium & 10 & 400 & $5.2^{c}$ & $0.52^{\mathrm{c}}$ & $\begin{array}{l}\text { EPA } 6010 \text { or } \\
200.8 \text { (ICP or } \\
\text { ICP/MS) }\end{array}$ \\
\hline Silver & 1 & 400 & 13.6 & 0.442 & $\begin{array}{l}\text { EPA } 6010 \text { or } \\
200.8 \text { (ICP or } \\
\text { ICP/MS) }\end{array}$ \\
\hline Thallium & 5 & 5.6 & 1.59 & 4.46 & $\begin{array}{l}\text { EPA } 6010 \text { or } \\
200.8 \text { (ICP or } \\
\text { ICP/MS) }\end{array}$ \\
\hline Vanadium & 2.5 & 560 & 2,240 & NV & $\begin{array}{l}\text { EPA } 6010 \text { or } \\
200.8 \text { (ICP or } \\
\text { ICP/MS) }\end{array}$ \\
\hline Zinc & 1 & 24,000 & 5,970 & 226 & $\begin{array}{l}\text { EPA } 6010 \text { or } \\
200.8 \text { (ICP or } \\
\text { ICP/MS) }\end{array}$ \\
\hline Chromium (hexavalent) & 0.5 & TBD & TBD & TBD & $\begin{array}{l}\text { EPA } 7196 \\
\text { (hexavalent } \\
\text { chromium) }\end{array}$ \\
\hline
\end{tabular}

${ }^{a}$ Units are $\mathrm{mg} / \mathrm{kg}$ (nonradionuclides) and pCi/g (radionuclides) unless otherwise noted. Cleanup levels are established in the most current CLARC table (updated February 12, 2009) calculated per Washington Administrative Code 173-340 (Ecology 2007) using input parameters stated in the CLARC table.

b Where cleanup levels are less than background, cleanup levels default to background as discussed in Section 2.1.2.1 of the 100 Area RDR/RAWP (DOE-RL-96-17, Rev 5).

c Where cleanup levels are less than PQLs, cleanup levels default to PQLs as discussed in Section 2.1.2.1 of the 100 Area RDR/RAWP (DOE-RL-96-17, Rev 5).

Reference: Washington State Department of Ecology, 2007, "Model Toxics Control Act Statute and Regulation," Publication No. 94-06, revised November 2007, Washington State Department of Ecology, Olympia, Washington.

CLARC = Cleanup Levels and Risk Calculation MS = mass spectroscopy

$\mathrm{EPA} \quad=$ U.S. Environmental Protection Agency $\mathrm{PQL} \quad=$ practical quantitation limit

GEA = gamma energy analysis

$\mathrm{ICP} \quad=$ inductively coupled plasma

RDR/RAWP $=$ remedial design/remedial action work plan

TBD $\quad=$ to be determined

NV = No value. The generic RESidual RADioactivity modeling reported in the 100 Area RDR/RAWP predicts the contaminant will not reach groundwater within 1,000 years.

Table 8. Wells \#3, \#4, and \#5 Target Analytes, Analytical Methods, and Contract-Required Detection Limits. (9 Pages)

\begin{tabular}{|c|c|c|c|c|c|}
\hline \multirow{2}{*}{ Target Analyte } & \multirow{2}{*}{$\begin{array}{c}\text { Practical } \\
\text { Quantitation } \\
\text { Limits }^{a}\end{array}$} & \multicolumn{3}{|c|}{ Preliminary Cleanup Goals ${ }^{a, b}$} & \multirow{2}{*}{$\begin{array}{l}\text { Analytical } \\
\text { Methods }\end{array}$} \\
\hline & & $\begin{array}{c}\text { Direct } \\
\text { Exposure }\end{array}$ & $\begin{array}{c}\text { Groundwater } \\
\text { Protection }\end{array}$ & $\begin{array}{c}\text { River } \\
\text { Protection }\end{array}$ & \\
\hline \multicolumn{6}{|c|}{ Radionuclides } \\
\hline Americium-241 ${ }^{\mathrm{C}}$ & 1 & 31.1 & NV & NV & $\begin{array}{l}\mathrm{Am}-241 / \\
\mathrm{Cm}-244 \mathrm{AEA}\end{array}$ \\
\hline Curium-243 & 1 & 22.1 & NV & NV & $\begin{array}{l}\text { Am-241/ } \\
\mathrm{Cm}-244 \text { AEA }\end{array}$ \\
\hline Cesium-137 & 0.1 & 6.2 & 1,465 & 2,930 & GEA \\
\hline
\end{tabular}


WCH-331

Rev. 0

Table 8. Wells \#3, \#4, and \#5 Target Analytes, Analytical Methods, and Contract-Required Detection Limits. (9 Pages)

\begin{tabular}{|c|c|c|c|c|c|}
\hline \multirow[b]{2}{*}{ Target Analyte } & \multirow{2}{*}{$\begin{array}{c}\text { Practical } \\
\text { Quantitation } \\
\text { Limits }^{a}\end{array}$} & \multicolumn{3}{|c|}{ Preliminary Cleanup Goals ${ }^{a, b}$} & \multirow{2}{*}{$\begin{array}{l}\text { Analytical } \\
\text { Methods }\end{array}$} \\
\hline & & $\begin{array}{c}\text { Direct } \\
\text { Exposure }\end{array}$ & $\begin{array}{c}\text { Groundwater } \\
\text { Protection }\end{array}$ & $\begin{array}{c}\text { River } \\
\text { Protection }\end{array}$ & \\
\hline Cobalt-60 & 0.05 & 1.4 & 13,900 & 27,800 & GEA \\
\hline Europium-152 & 0.1 & 3.3 & NV & NV & GEA \\
\hline Europium-154 & 0.1 & 3.0 & NV & NV & GEA \\
\hline Europium-155 & 0.1 & 125 & NV & NV & GEA \\
\hline Niobium-94 & 0.2 & 2.43 & NV & NV & GEA \\
\hline Silver-108m & 0.2 & 2.38 & NV & NV & GEA \\
\hline Strontium-90 & 1 & 4.5 & 27.6 & 55.2 & $\begin{array}{l}\text { Gas flow } \\
\text { proportional } \\
\text { counting }\end{array}$ \\
\hline lodine-129 & 2 & $2^{b}$ & $2^{b}$ & $2^{b}$ & $\begin{array}{l}\text { Low energy } \\
\text { GEA }\end{array}$ \\
\hline Plutonium-238 & 1 & 35.1 & NV & NV & \multirow{2}{*}{$\begin{array}{l}\text { Isotopic Pu } \\
\text { AEA }\end{array}$} \\
\hline Plutonium-239/240 & 1 & 33.9 & NV & NV & \\
\hline Uranium-233/234 & 1 & $1.1^{b}$ & $1.1^{b}$ & $1.1^{b}$ & $\begin{array}{l}\text { Isotopic U } \\
\text { AEA }\end{array}$ \\
\hline Uranium-235 & 1 & 0.61 & $0.185^{d}$ & $0.185^{d}$ & $\begin{array}{l}\text { Isotopic U } \\
\text { AEA }\end{array}$ \\
\hline Uranium-238 & 1 & $1.1^{b}$ & $1.1^{b}$ & $1.1^{b}$ & $\begin{array}{l}\text { Isotopic U } \\
\text { AEA }\end{array}$ \\
\hline Carbon-14 & 2 & 8.7 & NV & NV & $\begin{array}{l}\text { Liquid } \\
\text { scintillation } \\
\text { counting }\end{array}$ \\
\hline Nickel-63 & 30 & 4,026 & 83 & 166 & $\begin{array}{l}\text { Liquid } \\
\text { scintillation } \\
\text { counting }\end{array}$ \\
\hline Technetium-99 & 0.25 & 5.7 & 0.46 & 0.46 & $\begin{array}{l}\text { Liquid } \\
\text { scintillation } \\
\text { counting }\end{array}$ \\
\hline Tritium & 10 & 510 & 12.6 & 25.2 & $\begin{array}{l}\text { Liquid } \\
\text { scintillation } \\
\text { counting }\end{array}$ \\
\hline Neptunium-237 & 1 & 2.44 & $0.9^{d}$ & $1.80^{d}$ & Np-237 AEA \\
\hline \multicolumn{6}{|c|}{ Nonradionuclides } \\
\hline Chloride & 2 & NV & 25,000 & NA & $\begin{array}{l}\text { EPA } 300.0 \\
\text { (anions by IC) }\end{array}$ \\
\hline Fluoride & 5 & 4,800 & 2,800 & 2,890 & $\begin{array}{l}\text { EPA } 300.0 \\
\text { (anions by IC) }\end{array}$ \\
\hline Nitrate (as nitrogen) & 2.5 & 128,000 & 40 & 40 & $\begin{array}{l}\text { EPA } 300.0 \\
\text { (anions by IC) }\end{array}$ \\
\hline Nitrite (as nitrogen) & 2.5 & 8,000 & 40 & 4 & $\begin{array}{l}\text { EPA } 300.0 \\
\text { (anions by IC) }\end{array}$ \\
\hline Sulfate & 5 & NV & 1,030 & 1,030 & $\begin{array}{l}\text { EPA } 300.0 \\
\text { (anions by IC) }\end{array}$ \\
\hline
\end{tabular}


Table 8. Wells \#3, \#4, and \#5 Target Analytes, Analytical Methods, and Contract-Required Detection Limits. (9 Pages)

\begin{tabular}{|c|c|c|c|c|c|}
\hline \multirow[b]{2}{*}{ Target Analyte } & \multirow{2}{*}{$\begin{array}{c}\text { Practical } \\
\text { Quantitation } \\
\text { Limits }^{\mathrm{a}}\end{array}$} & \multicolumn{3}{|c|}{ Preliminary Cleanup Goals ${ }^{a, b}$} & \multirow{2}{*}{$\begin{array}{l}\text { Analytical } \\
\text { Methods }\end{array}$} \\
\hline & & $\begin{array}{c}\text { Direct } \\
\text { Exposure }\end{array}$ & $\begin{array}{c}\text { Groundwater } \\
\text { Protection }\end{array}$ & $\begin{array}{c}\text { River } \\
\text { Protection }\end{array}$ & \\
\hline Aluminum & 5 & 80,000 & 480,000 & 480,000 & $\begin{array}{l}\text { EPA } 6010 \text { or } \\
200.8 \text { (ICP or } \\
\text { ICP/MS) }\end{array}$ \\
\hline Antimony & 6 & 32 & 5.4 & 12.7 & $\begin{array}{l}\text { EPA } 6010 \text { or } \\
200.8 \text { (ICP or } \\
\text { ICP/MS) }\end{array}$ \\
\hline Arsenic & 10 & $\mathrm{TBD}^{\mathrm{b}}$ & $\mathrm{TBD}^{\mathrm{b}}$ & $\mathrm{TBD}^{\mathrm{b}}$ & $\begin{array}{l}\text { EPA } 6010 \text { or } \\
200.8 \text { (ICP or } \\
\text { ICP/MS) }\end{array}$ \\
\hline Barium & 2 & 16,000 & 1,650 & 1,600 & $\begin{array}{l}\text { EPA } 6010 \text { or } \\
200.8 \text { (ICP or } \\
\text { ICP/MS) }\end{array}$ \\
\hline Beryllium & 0.5 & 160 & 63.2 & 63 & $\begin{array}{l}\text { EPA } 6010 \text { or } \\
200.8 \text { (ICP or } \\
\text { ICP/MS) }\end{array}$ \\
\hline Boron & 2 & 16,000 & 210 & NV & $\begin{array}{l}\text { EPA } 6010 \text { or } \\
200.8 \text { (ICP or } \\
\text { ICP/MS) }\end{array}$ \\
\hline Cadmium & 0.5 & 80 & 0.69 & $0.125^{d}$ & $\begin{array}{l}\text { EPA } 6010 \text { or } \\
200.8 \text { (ICP or } \\
\text { ICP/MS) }\end{array}$ \\
\hline Chromium (total) & 1 & 120,000 & 2,000 & 1,300 & $\begin{array}{l}\text { EPA } 6010 \text { or } \\
200.8 \text { (ICP or } \\
\text { ICP/MS) }\end{array}$ \\
\hline Cobalt & 2 & 24 & 15.7 & NV & $\begin{array}{l}\text { EPA } 6010 \text { or } \\
200.8 \text { (ICP or } \\
\text { ICP/MS) }\end{array}$ \\
\hline Copper & 1 & 3,200 & 284 & 575 & $\begin{array}{l}\text { EPA } 6010 \text { or } \\
200.8 \text { (ICP or } \\
\text { ICP/MS) }\end{array}$ \\
\hline Lead & 5 & 250 & 3,000 & 420 & $\begin{array}{l}\text { EPA } 6010 \text { or } \\
200.8 \text { (ICP or } \\
\text { ICP/MS) }\end{array}$ \\
\hline Lithium & 2.5 & 160 & 192 & NV & $\begin{array}{l}\text { EPA } 6010 \text { or } \\
200.8 \text { (ICP or } \\
\text { ICP/MS) }\end{array}$ \\
\hline Manganese & 5 & 3,760 & $512^{b}$ & $256^{b}$ & $\begin{array}{l}\text { EPA } 6010 \text { or } \\
200.8 \text { (ICP or } \\
\text { ICP/MS) }\end{array}$ \\
\hline Molybdenum & 2 & 400 & 32 & NV & $\begin{array}{l}\text { EPA } 6010 \text { or } \\
200.8 \text { (ICP or } \\
\text { ICP/MS) }\end{array}$ \\
\hline Nickel & 4 & 1,600 & 130 & 179 & $\begin{array}{l}\text { EPA } 6010 \text { or } \\
200.8 \text { (ICP or } \\
\text { ICP/MS) }\end{array}$ \\
\hline Selenium & 10 & 400 & $5.2^{d}$ & $0.52^{d}$ & $\begin{array}{l}\text { EPA } 6010 \text { or } \\
200.8 \text { (ICP or } \\
\text { ICP/MS) }\end{array}$ \\
\hline
\end{tabular}


Table 8. Wells \#3, \#4, and \#5 Target Analytes, Analytical Methods, and Contract-Required Detection Limits. (9 Pages)

\begin{tabular}{|c|c|c|c|c|c|}
\hline \multirow[b]{2}{*}{ Target Analyte } & \multirow{2}{*}{$\begin{array}{c}\text { Practical } \\
\text { Quantitation } \\
\text { Limits }^{a}\end{array}$} & \multicolumn{3}{|c|}{ Preliminary Cleanup Goals $^{a, b}$} & \multirow{2}{*}{$\begin{array}{l}\text { Analytical } \\
\text { Methods }\end{array}$} \\
\hline & & $\begin{array}{c}\text { Direct } \\
\text { Exposure }\end{array}$ & $\begin{array}{c}\text { Groundwater } \\
\text { Protection }\end{array}$ & $\begin{array}{c}\text { River } \\
\text { Protection }\end{array}$ & \\
\hline Silver & 1 & 400 & 13.6 & 0.442 & $\begin{array}{l}\text { EPA } 6010 \text { or } \\
200.8 \text { (ICP or } \\
\text { ICP/MS) }\end{array}$ \\
\hline Thallium & 5 & 5.6 & 1.59 & 4.46 & $\begin{array}{l}\text { EPA } 6010 \text { or } \\
200.8 \text { (ICP or } \\
\text { ICP/MS) }\end{array}$ \\
\hline Vanadium & 2.5 & 560 & 2,240 & NV & $\begin{array}{l}\text { EPA } 6010 \text { or } \\
200.8 \text { (ICP or } \\
\text { ICP/MS) }\end{array}$ \\
\hline Zinc & 1 & 24,000 & 5,970 & 226 & $\begin{array}{l}\text { EPA } 6010 \text { or } \\
200.8 \text { (ICP or } \\
\text { ICP/MS) }\end{array}$ \\
\hline Chromium (hexavalent) & 0.5 & TBD & TBD & TBD & $\begin{array}{l}\text { EPA } 7196 \\
\text { (hexavalent } \\
\text { chromium) }\end{array}$ \\
\hline Mercury & 0.2 & 24 & 2.09 & $0.33^{b}$ & $\begin{array}{l}\text { EPA } 7471(\mathrm{Hg} \\
\text { cold vapor) }\end{array}$ \\
\hline Ethylene glycol & 5.0 & 160,000 & 64.3 & NV & EPA 8015 \\
\hline Propylene glycol & 5 & $1,600,000$ & NV & NV & EPA 8015 \\
\hline Methanol & 1.0 & 4,000 & 16.1 & NV & EPA $8015 M$ \\
\hline Aldrin & 0.00165 & 0.0588 & 0.00504 & $0.0008^{d}$ & $\begin{array}{l}\text { EPA } 8081 \\
\text { (pesticides) }\end{array}$ \\
\hline BHC; alpha & 0.00165 & 0.159 & $0.0005^{d}$ & $0.00031^{d}$ & $\begin{array}{l}\text { EPA } 8081 \\
\text { (pesticides) }\end{array}$ \\
\hline $\mathrm{BHC}$; beta & 0.00165 & 0.556 & 0.00227 & 0.00259 & $\begin{array}{l}\text { EPA } 8081 \\
\text { (pesticides) }\end{array}$ \\
\hline $\mathrm{BHC}$; delta & 0.00165 & NV & NV & NV & $\begin{array}{l}\text { EPA 8081 } \\
\text { (pesticides) }\end{array}$ \\
\hline BHC; gamma (lindane) & 0.00165 & 0.769 & 0.00209 & 0.00119 & $\begin{array}{l}\text { EPA } 8081 \\
\text { (pesticides) }\end{array}$ \\
\hline Chlordane & 0.0165 & 2.86 & 2.06 & $0.00055^{d}$ & $\begin{array}{l}\text { EPA 8081 } \\
\text { (pesticides) }\end{array}$ \\
\hline DDD; 4,4'- & 0.0033 & 4.17 & 0.335 & $0.00024^{d}$ & $\begin{array}{l}\text { EPA } 8081 \\
\text { (pesticides) }\end{array}$ \\
\hline DDE; 4,4'- & 0.0033 & 2.94 & 0.446 & $0.006^{d}$ & $\begin{array}{l}\text { EPA } 8081 \\
\text { (pesticides) }\end{array}$ \\
\hline DDT; 4,4'- & 0.0033 & 2.94 & 3.49 & 0.00493 & $\begin{array}{l}\text { EPA } 8081 \\
\text { (pesticides) }\end{array}$ \\
\hline Dieldrin & 0.0033 & 0.0625 & $0.0028^{d}$ & $0.00004^{d}$ & $\begin{array}{l}\text { EPA } 8081 \\
\text { (pesticides) }\end{array}$ \\
\hline Endosulfan I & 0.00165 & 480 & 4.3 & 0.0417 & $\begin{array}{l}\text { EPA 8081 } \\
\text { (pesticides) }\end{array}$ \\
\hline Endosulfan II & 0.0033 & 480 & 4.3 & 0.0417 & $\begin{array}{l}\text { EPA } 8081 \\
\text { (pesticides) }\end{array}$ \\
\hline Endosulfan sulfate & 0.0033 & 480 & 4.3 & 0.0417 & $\begin{array}{l}\text { EPA } 8081 \\
\text { (pesticides) }\end{array}$ \\
\hline
\end{tabular}


Table 8. Wells \#3, \#4, and \#5 Target Analytes, Analytical Methods, and Contract-Required Detection Limits. (9 Pages)

\begin{tabular}{|c|c|c|c|c|c|}
\hline \multirow[b]{2}{*}{ Target Analyte } & \multirow{2}{*}{$\begin{array}{c}\text { Practical } \\
\text { Quantitation } \\
\text { Limits }^{a}\end{array}$} & \multicolumn{3}{|c|}{ Preliminary Cleanup Goals ${ }^{a, b}$} & \multirow{2}{*}{$\begin{array}{l}\text { Analytical } \\
\text { Methods }\end{array}$} \\
\hline & & $\begin{array}{c}\text { Direct } \\
\text { Exposure }\end{array}$ & $\begin{array}{l}\text { Groundwater } \\
\text { Protection }\end{array}$ & $\begin{array}{c}\text { River } \\
\text { Protection }\end{array}$ & \\
\hline Endrin & 0.0033 & 24 & 0.44 & 0.168 & $\begin{array}{l}\text { EPA } 8081 \\
\text { (pesticides) }\end{array}$ \\
\hline Endrin aldehyde & 0.0033 & 24 & 0.44 & 0.168 & $\begin{array}{l}\text { EPA } 8081 \\
\text { (pesticides) }\end{array}$ \\
\hline Endrin ketone & 0.0033 & 24 & 0.44 & 0.168 & $\begin{array}{l}\text { EPA } 8081 \\
\text { (pesticides) }\end{array}$ \\
\hline Heptachlor & 0.00165 & 0.222 & 0.0370 & $0.000025^{d}$ & $\begin{array}{l}\text { EPA } 8081 \\
\text { (pesticides) }\end{array}$ \\
\hline Heptachlor epoxide & 0.00165 & 0.11 & 0.008 & $0.0001^{d}$ & $\begin{array}{l}\text { EPA } 8081 \\
\text { (pesticides) }\end{array}$ \\
\hline Methoxychlor & 0.0165 & 400 & 64.2 & 13.4 & $\begin{array}{l}\text { EPA } 8081 \\
\text { (pesticides) }\end{array}$ \\
\hline Toxaphene & 0.165 & 0.909 & 0.153 & 0.0005 & $\begin{array}{l}\text { EPA } 8081 \\
\text { (pesticides) }\end{array}$ \\
\hline Aroclor-1016 (PCB) & 0.0165 & 0.5 & 0.094 & $0.000224^{d}$ & $\begin{array}{l}\text { EPA } 8082 \\
\text { (PCBs) }\end{array}$ \\
\hline Aroclor-1221 (PCB) & 0.0165 & 0.5 & $0.00920^{d}$ & $0.0000219^{d}$ & $\begin{array}{l}\text { EPA 8082 } \\
\text { (PCBs) }\end{array}$ \\
\hline Aroclor-1232 (PCB) & 0.0165 & 0.5 & $0.00920^{d}$ & $0.0000219^{d}$ & $\begin{array}{l}\text { EPA 8082 } \\
\text { (PCBs) }\end{array}$ \\
\hline Aroclor-1242 (PCB) & 0.0165 & 0.5 & 0.0394 & $0.0000935^{d}$ & $\begin{array}{l}\text { EPA } 8082 \\
\text { (PCBs) }\end{array}$ \\
\hline Aroclor-1248 (PCB) & 0.0165 & 0.5 & 0.0386 & $0.0000935^{d}$ & $\begin{array}{l}\text { EPA } 8082 \\
\text { (PCBs) }\end{array}$ \\
\hline Aroclor-1254 (PCB) & 0.0165 & 0.5 & 0.0664 & $0.000158^{d}$ & $\begin{array}{l}\text { EPA } 8082 \\
\text { (PCBs) }\end{array}$ \\
\hline Aroclor-1260 (PCB) & 0.0165 & 0.5 & 0.721 & $0.00171^{d}$ & $\begin{array}{l}\text { EPA } 8082 \\
\text { (PCBs) }\end{array}$ \\
\hline Aroclor-1262 (PCB) & 0.0165 & NV & NV & NV & $\begin{array}{l}\text { EPA } 8082 \\
\text { (PCBs) }\end{array}$ \\
\hline Dalapon & 0.1 & 2,400 & 0.811 & 0.81 & $\begin{array}{l}\text { EPA } 8151 \\
\text { (herbicides) }\end{array}$ \\
\hline $\mathrm{DB} ; 2,4-$ & 0.1 & 640 & 0.768 & NV & $\begin{array}{l}\text { EPA } 8151 \\
\text { (herbicides) }\end{array}$ \\
\hline Dicamba & 0.1 & 2,400 & 2.2 & 2.20 & $\begin{array}{l}\text { EPA } 8151 \\
\text { (herbicides) }\end{array}$ \\
\hline $\begin{array}{l}\text { Dichlorophenoxyacetic } \\
\text { acid; } 2,4-\end{array}$ & 0.4 & 800 & $0.321^{d}$ & 0.321 & $\begin{array}{l}\text { EPA } 8151 \\
\text { (herbicides) }\end{array}$ \\
\hline Dichlorprop & 0.1 & 640 & 0.321 & 0.321 & $\begin{array}{l}\text { EPA } 8151 \\
\text { (herbicides) }\end{array}$ \\
\hline $\begin{array}{l}\text { Dinoseb (DNBP) }\{2 \text {-sec- } \\
\text { butyl-4,6-dinitrophenol\} }\end{array}$ & 0.012 & 80 & 0.0524 & 1.05 & $\begin{array}{l}\text { EPA } 8151 \\
\text { (herbicides) }\end{array}$ \\
\hline TP; 2,4,5- (Silvex) & 0.02 & 640 & 0.28 & 0.28 & $\begin{array}{l}\text { EPA 8151 } \\
\text { (herbicides) }\end{array}$ \\
\hline $\begin{array}{l}\text { Trichlorophenoxyacetic } \\
\text { acid; } 2,4,5-\end{array}$ & 0.02 & 800 & 0.761 & 0.79 & $\begin{array}{l}\text { EPA } 8151 \\
\text { (herbicides) }\end{array}$ \\
\hline Acetone & 0.02 & 72,000 & 28.9 & NV & $\begin{array}{l}\text { EPA } 8260 \\
\text { (VOCs) }\end{array}$ \\
\hline
\end{tabular}


Table 8. Wells \#3, \#4, and \#5 Target Analytes, Analytical Methods, and Contract-Required Detection Limits. (9 Pages)

\begin{tabular}{|c|c|c|c|c|c|}
\hline \multirow[b]{2}{*}{ Target Analyte } & \multirow{2}{*}{$\begin{array}{c}\text { Practical } \\
\text { Quantitation } \\
\text { Limits }^{a}\end{array}$} & \multicolumn{3}{|c|}{ Preliminary Cleanup Goals ${ }^{a, b}$} & \multirow{2}{*}{$\begin{array}{l}\text { Analytical } \\
\text { Methods }\end{array}$} \\
\hline & & $\begin{array}{c}\text { Direct } \\
\text { Exposure }\end{array}$ & $\begin{array}{l}\text { Groundwater } \\
\text { Protection }\end{array}$ & $\begin{array}{c}\text { River } \\
\text { Protection }\end{array}$ & \\
\hline Benzene & 0.005 & 18.2 & 0.004 & 0.007 & $\begin{array}{l}\text { EPA } 8260 \\
\text { (VOCs) }\end{array}$ \\
\hline Butanol; 1- & 0.1 & 8,000 & 3.31 & 6.6 & $\begin{array}{l}\text { EPA } 8260 \\
\text { (VOCs) }\end{array}$ \\
\hline Butanone; 2- & 0.01 & 48,000 & 19.6 & NV & $\begin{array}{l}\text { EPA } 8260 \\
\text { (VOCs) }\end{array}$ \\
\hline Carbon disulfide & 0.005 & 800 & 5.65 & 12.4 & $\begin{array}{l}\text { EPA } 8260 \\
\text { (VOCs) }\end{array}$ \\
\hline Carbon tetrachloride & 0.005 & 7.69 & 0.031 & $0.0023^{d}$ & $\begin{array}{l}\text { EPA } 8260 \\
\text { (VOCs) }\end{array}$ \\
\hline Chloroform & 0.005 & 164 & 0.038 & 0.0304 & $\begin{array}{l}\text { EPA } 8260 \\
\text { (VOCs) }\end{array}$ \\
\hline Cyclohexanone & 1 & 400,000 & 172 & NV & $\begin{array}{l}\text { EPA } 8260 \\
\text { (VOCs) }\end{array}$ \\
\hline Ethyl acetate & 5 & NV & 29.8 & NV & $\begin{array}{l}\text { EPA } 8260 \\
\text { (VOCs) }\end{array}$ \\
\hline Ethyl ether & 0.005 & 16,000 & 6.68 & NV & $\begin{array}{l}\text { EPA } 8260 \\
\text { (VOCs) }\end{array}$ \\
\hline Ethylbenzene & 0.005 & 8,000 & 6.05 & 26.8 & $\begin{array}{l}\text { EPA } 8260 \\
\text { (VOCs) }\end{array}$ \\
\hline Methyl isobutyl ketone & 0.01 & 6,400 & 2.71 & NV & $\begin{array}{l}\text { EPA } 8260 \\
\text { (VOCs) }\end{array}$ \\
\hline Methylene chloride & 0.005 & 133 & 0.218 & 0.0205 & $\begin{array}{l}\text { EPA } 8260 \\
\text { (VOCs) }\end{array}$ \\
\hline Tetrachloroethene & 0.005 & 1.85 & 0.008 & 0.004 & $\begin{array}{l}\text { EPA } 8260 \\
\text { (VOCs) }\end{array}$ \\
\hline Toluene & 0.005 & 6,400 & 4.65 & 49.5 & $\begin{array}{l}\text { EPA } 8260 \\
\text { (VOCs) }\end{array}$ \\
\hline Trichlorobenzene; 1,2,4- & 0.01 & 800 & 2.98 & 1.31 & $\begin{array}{l}\text { EPA } 8260 \\
\text { (VOCs) }\end{array}$ \\
\hline Trichloroethane; 1,1,1- & 0.005 & NV & 1.58 & 1.59 & $\begin{array}{l}\text { EPA } 8260 \\
\text { (VOCs) }\end{array}$ \\
\hline Trichloroethene & 0.005 & 11.2 & 0.00323 & 0.0178 & $\begin{array}{l}\text { EPA } 8260 \\
\text { (VOCs) }\end{array}$ \\
\hline Vinyl chloride & 0.005 & 240 & 0.00184 & 0.0126 & $\begin{array}{l}\text { EPA } 8260 \\
\text { (VOCs) }\end{array}$ \\
\hline Xylenes (total) & 0.01 & 16,000 & 3.09 & 5.15 & $\begin{array}{l}\text { EPA } 8260 \\
\text { (VOCs) }\end{array}$ \\
\hline Tributyl phosphate & 3.3 & 185 & $0.677^{d}$ & NV & $\begin{array}{l}\text { EPA } 8270 \\
\text { (SVOCs) }\end{array}$ \\
\hline $\begin{array}{l}\text { Bis(2-chloro-1-methylethyl) } \\
\text { ether }\end{array}$ & 0.33 & 14.3 & NV & 0.180 & $\begin{array}{l}\text { EPA } 8270 \\
\text { (SVOCs) }\end{array}$ \\
\hline $\begin{array}{l}\text { Bis(2-chloroethoxy) } \\
\text { methane }\end{array}$ & 0.33 & 0.909 & NV & NV & $\begin{array}{l}\text { EPA } 8270 \\
\text { (SVOCs) }\end{array}$ \\
\hline Bis(2-ethylhexyl)phthalate & 0.33 & 71.4 & 13.9 & 4.01 & $\begin{array}{l}\text { EPA } 8270 \\
\text { (SVOCs) }\end{array}$ \\
\hline $\begin{array}{l}\text { Bromophenylphenyl } \\
\text { ether;4- }\end{array}$ & 0.33 & NV & NV & NV & $\begin{array}{l}\text { EPA } 8270 \\
\text { (SVOCs) }\end{array}$ \\
\hline
\end{tabular}


Table 8. Wells \#3, \#4, and \#5 Target Analytes, Analytical Methods, and Contract-Required Detection Limits. (9 Pages)

\begin{tabular}{|c|c|c|c|c|c|}
\hline \multirow[b]{2}{*}{ Target Analyte } & \multirow{2}{*}{$\begin{array}{c}\text { Practical } \\
\text { Quantitation } \\
\text { Limits }^{\text {a }}\end{array}$} & \multicolumn{3}{|c|}{ Preliminary Cleanup Goals ${ }^{a, b}$} & \multirow{2}{*}{$\begin{array}{l}\text { Analytical } \\
\text { Methods }\end{array}$} \\
\hline & & $\begin{array}{c}\text { Direct } \\
\text { Exposure }\end{array}$ & $\begin{array}{l}\text { Groundwater } \\
\text { Protection }\end{array}$ & $\begin{array}{c}\text { River } \\
\text { Protection }\end{array}$ & \\
\hline Butylbenzylphthalate & 0.33 & 16,000 & 893 & 349 & $\begin{array}{l}\text { EPA } 8270 \\
\text { (SVOCs) }\end{array}$ \\
\hline Carbazole & 0.33 & 50 & $0.314^{d}$ & NV & $\begin{array}{l}\text { EPA } 8270 \\
\text { (SVOCs) }\end{array}$ \\
\hline Chloro-3-methylphenol;4- & 0.33 & 4,000 & NV & NV & $\begin{array}{l}\text { EPA } 8270 \\
\text { (SVOCs) }\end{array}$ \\
\hline $\begin{array}{l}\text { Chlorophenylphenyl } \\
\text { ether;4- }\end{array}$ & 0.33 & 4,000 & NV & NV & $\begin{array}{l}\text { EPA } 8270 \\
\text { (SVOCs) }\end{array}$ \\
\hline Dibenzofuran & 0.33 & 160 & 7.36 & NV & $\begin{array}{l}\text { EPA } 8270 \\
\text { (SVOCs) }\end{array}$ \\
\hline Diethylphthalate & 0.33 & 64,000 & 72.2 & 130 & $\begin{array}{l}\text { EPA } 8270 \\
\text { (SVOCs) }\end{array}$ \\
\hline Di-n-butylphthalate & 0.33 & 8,000 & 56.5 & 95.5 & $\begin{array}{l}\text { EPA } 8270 \\
\text { (SVOCs) }\end{array}$ \\
\hline Di-n-octylphthalate & 0.33 & 1,600 & 532,000 & NV & $\begin{array}{l}\text { EPA } 8270 \\
\text { (SVOCs) }\end{array}$ \\
\hline Isophorone & 0.33 & 1,050 & $0.228^{d}$ & 7.7 & $\begin{array}{l}\text { EPA } 8270 \\
\text { (SVOCs) }\end{array}$ \\
\hline Methylnaphthalene; 2- & 0.33 & 320 & 2.03 & 2.04 & $\begin{array}{l}\text { EPA 8270 } \\
\text { (SVOCs) }\end{array}$ \\
\hline Methylphenol; 4-(cresol; p-) & 0.33 & 400 & 0.507 & 0.505 & $\begin{array}{l}\text { EPA } 8270 \\
\text { (SVOCs) }\end{array}$ \\
\hline Chloroaniline;4- & 0.33 & 320 & $0.264^{d}$ & NV & $\begin{array}{l}\text { EPA } 8270 \\
\text { (SVOCs) }\end{array}$ \\
\hline Chloronaphthalene;2- & 0.33 & 6,400 & 40.7 & 56.5 & $\begin{array}{l}\text { EPA } 8270 \\
\text { (SVOCs) }\end{array}$ \\
\hline Chlorophenol;2- & 0.33 & 400 & 0.472 & 2.28 & $\begin{array}{l}\text { EPA } 8270 \\
\text { (SVOCs) }\end{array}$ \\
\hline Dichlorobenzene; 1,2- & 0.33 & 7,200 & 7.03 & 31.7 & $\begin{array}{l}\text { EPA } 8270 \\
\text { (SVOCs) }\end{array}$ \\
\hline Dichlorobenzene; 1,3- & 0.33 & 2,400 & 3.09 & 5.15 & $\begin{array}{l}\text { EPA } 8270 \\
\text { (SVOCs) }\end{array}$ \\
\hline Dichlorobenzene; 1,4- & 0.33 & 41.7 & $0.030^{d}$ & $0.080^{d}$ & $\begin{array}{l}\text { EPA } 8270 \\
\text { (SVOCs) }\end{array}$ \\
\hline Dichlorobenzidine; 3,3'- & 0.33 & 2.22 & $0.003^{d}$ & $0.0005^{d}$ & $\begin{array}{l}\text { EPA 8270 } \\
\text { (SVOCs) }\end{array}$ \\
\hline Dichlorophenol; 2,4- & 0.33 & 240 & NV & 2.65 & $\begin{array}{l}\text { EPA } 8270 \\
\text { (SVOCs) }\end{array}$ \\
\hline Dimethylphenol; 2,4- & 0.33 & 1,600 & 2.62 & 4.52 & $\begin{array}{l}\text { EPA } 8270 \\
\text { (SVOCs) }\end{array}$ \\
\hline Dimethylphthalate & 0.33 & 64,000 & 72.2 & 130 & $\begin{array}{l}\text { EPA } 8270 \\
\text { (SVOCs) }\end{array}$ \\
\hline $\begin{array}{l}\text { Di-nitro-2-methylphenol; } \\
4,6 \text { - }\end{array}$ & 0.33 & 8.00 & $0.0256^{d}$ & NV & $\begin{array}{l}\text { EPA 8270 } \\
\text { (SVOCs) }\end{array}$ \\
\hline Dinitrophenol; 2,4- & 0.825 & 160 & $0.125^{d}$ & $0.560^{d}$ & $\begin{array}{l}\text { EPA } 8270 \\
\text { (SVOCs) }\end{array}$ \\
\hline Dinitrotoluene; 2,4- & 0.33 & 160 & $0.189^{d}$ & $0.0005^{d}$ & $\begin{array}{l}\text { EPA } 8270 \\
\text { (SVOCs) }\end{array}$ \\
\hline
\end{tabular}


Table 8. Wells \#3, \#4, and \#5 Target Analytes, Analytical Methods, and Contract-Required Detection Limits. (9 Pages)

\begin{tabular}{|c|c|c|c|c|c|}
\hline \multirow[b]{2}{*}{ Target Analyte } & \multirow{2}{*}{$\begin{array}{c}\text { Practical } \\
\text { Quantitation } \\
\text { Limits }^{a}\end{array}$} & \multicolumn{3}{|c|}{ Preliminary Cleanup Goals ${ }^{a, b}$} & \multirow{2}{*}{$\begin{array}{l}\text { Analytical } \\
\text { Methods }\end{array}$} \\
\hline & & $\begin{array}{c}\text { Direct } \\
\text { Exposure }\end{array}$ & $\begin{array}{c}\text { Groundwater } \\
\text { Protection }\end{array}$ & $\begin{array}{c}\text { River } \\
\text { Protection }\end{array}$ & \\
\hline Dinitrotoluene; 2,6- & 0.33 & 80 & NV & NV & $\begin{array}{l}\text { EPA } 8270 \\
\text { (SVOCs) }\end{array}$ \\
\hline Hexachlorobenzene & 0.33 & 0.625 & $0.015^{d}$ & $0.001^{d}$ & $\begin{array}{l}\text { EPA } 8270 \\
\text { (SVOCs) }\end{array}$ \\
\hline Hexachlorobutadiene & 0.33 & 12.8 & 0.605 & 0.475 & $\begin{array}{l}\text { EPA 8270 } \\
\text { (SVOCs) }\end{array}$ \\
\hline Hexachlorocyclopentadiene & 0.33 & 480 & NV & 960 & $\begin{array}{l}\text { EPA } 8270 \\
\text { (SVOCs) }\end{array}$ \\
\hline Hexachloroethane & 0.33 & 71.4 & 0.125 & 0.076 & $\begin{array}{l}\text { EPA } 8270 \\
\text { (SVOCs) }\end{array}$ \\
\hline Methylphenol; 2-(cresol; o-) & 0.33 & 4,000 & 5.14 & 5.15 & $\begin{array}{l}\text { EPA } 8270 \\
\text { (SVOCs) }\end{array}$ \\
\hline Nitroaniline; 2- & 0.33 & 240 & 0.121 & NV & $\begin{array}{l}\text { EPA } 8270 \\
\text { (SVOCs) }\end{array}$ \\
\hline Nitroaniline; 3- & 0.33 & 47.6 & 0.010 & NV & $\begin{array}{l}\text { EPA } 8270 \\
\text { (SVOCs) }\end{array}$ \\
\hline Nitroaniline; 4- & 0.33 & 47.6 & 0.010 & NV & $\begin{array}{l}\text { EPA 8270 } \\
\text { (SVOCs) }\end{array}$ \\
\hline Nitrobenzene & 0.33 & 160 & $0.102^{d}$ & $0.109^{d}$ & $\begin{array}{l}\text { EPA } 8270 \\
\text { (SVOCs) }\end{array}$ \\
\hline Nitrophenol; 2- & 0.66 & NV & NV & NV & $\begin{array}{l}\text { EPA } 8270 \\
\text { (SVOCs) }\end{array}$ \\
\hline Nitrophenol; 4- & 0.66 & 640 & 1.30 & 128 & $\begin{array}{l}\text { EPA } 8270 \\
\text { (SVOCs) }\end{array}$ \\
\hline $\begin{array}{l}\text { Nitroso-di-n-propylamine; } \\
\mathrm{N} \text { - }\end{array}$ & 0.33 & $0.143^{d}$ & 0.000056 & 0.0035 & $\begin{array}{l}\text { EPA } 8270 \\
\text { (SVOCs) }\end{array}$ \\
\hline Nitrosodiphenylamine; N- & 0.33 & 204 & NV & NV & $\begin{array}{l}\text { EPA } 8270 \\
\text { (SVOCs) }\end{array}$ \\
\hline Pentachlorophenol & 0.33 & 8.33 & $0.016^{d}$ & $0.00444^{d}$ & $\begin{array}{l}\text { EPA } 8270 \\
\text { (SVOCs) }\end{array}$ \\
\hline Trichlorophenol; 2,4,5- & 0.33 & 8,000 & 28.8 & NV & $\begin{array}{l}\text { EPA } 8270 \\
\text { (SVOCs) }\end{array}$ \\
\hline Trichlorophenol; 2,4,6- & 0.33 & 90.9 & $0.0462^{d}$ & $0.049^{d}$ & $\begin{array}{l}\text { EPA } 8270 \\
\text { (SVOCs) }\end{array}$ \\
\hline Acenaphthene & 0.1 & 4,800 & 97.9 & 65.5 & $\begin{array}{l}\text { EPA } 8310 \\
\text { (PAHs) }\end{array}$ \\
\hline Acenaphthylene & 0.1 & 4,800 & 97.9 & 65.5 & $\begin{array}{l}\text { EPA } 8310 \\
\text { (PAHs) }\end{array}$ \\
\hline Anthracene & 0.05 & 24,000 & 2.270 & 4,550 & $\begin{array}{l}\text { EPA } 8310 \\
\text { (PAHs) }\end{array}$ \\
\hline Benzo(a)anthracene & 0.015 & 1.37 & 0.856 & 0.02400 & $\begin{array}{l}\text { EPA } 8310 \\
\text { (PAHs) }\end{array}$ \\
\hline Benzo(a)pyrene & 0.015 & 0.137 & 2.33 & 0.0545 & $\begin{array}{l}\text { EPA } 8310 \\
\text { (PAHs) }\end{array}$ \\
\hline Benzo(b)fluoranthene & 0.015 & 1.37 & 2.95 & 0.069 & $\begin{array}{l}\text { EPA 8310 } \\
\text { (PAHS) }\end{array}$ \\
\hline Benzo(ghi)perylene & 0.03 & 2,400 & 25,700 & 3,540 & $\begin{array}{l}\text { EPA } 8310 \\
\text { (PAHs) }\end{array}$ \\
\hline
\end{tabular}


Table 8. Wells \#3, \#4, and \#5 Target Analytes, Analytical Methods, and Contract-Required Detection Limits. (9 Pages)

\begin{tabular}{|c|c|c|c|c|c|}
\hline \multirow[b]{2}{*}{ Target Analyte } & \multirow{2}{*}{$\begin{array}{c}\text { Practical } \\
\text { Quantitation } \\
\text { Limits }^{a}\end{array}$} & \multicolumn{3}{|c|}{ Preliminary Cleanup Goals ${ }^{a, b}$} & \multirow{2}{*}{$\begin{array}{l}\text { Analytical } \\
\text { Methods }\end{array}$} \\
\hline & & $\begin{array}{c}\text { Direct } \\
\text { Exposure }\end{array}$ & $\begin{array}{c}\text { Groundwater } \\
\text { Protection }\end{array}$ & $\begin{array}{c}\text { River } \\
\text { Protection }\end{array}$ & \\
\hline Benzo(k)fluoranthene & 0.015 & 1.37 & 21.5 & 0.069 & $\begin{array}{l}\text { EPA } 8310 \\
\text { (PAHs) }\end{array}$ \\
\hline Chrysene & 0.1 & 13.7 & 9.56 & $0.0223^{d}$ & $\begin{array}{l}\text { EPA } 8310 \\
\text { (PAHs) }\end{array}$ \\
\hline Dibenz(a,h)anthracene & 0.3 & 1.37 & 4.29 & 0.1 & $\begin{array}{l}\text { EPA } 8310 \\
\text { (PAHs) }\end{array}$ \\
\hline Fluoranthene & 0.05 & 3,200 & 631 & 89 & $\begin{array}{l}\text { EPA } 8310 \\
\text { (PAHs) }\end{array}$ \\
\hline Fluorene & 0.03 & 3,200 & 101 & 206 & $\begin{array}{l}\text { EPA } 8310 \\
\text { (PAHs) }\end{array}$ \\
\hline Indeno(1,2,3-cd)pyrene & 0.03 & 1.37 & 8.33 & 0.195 & $\begin{array}{l}\text { EPA } 8310 \\
\text { (PAHs) }\end{array}$ \\
\hline Naphthalene & 0.1 & 1,600 & 4.46 & 138 & $\begin{array}{l}\text { EPA } 8310 \\
\text { (PAHs) }\end{array}$ \\
\hline Phenanthrene & 0.05 & 24,000 & 1,140 & 9,100 & $\begin{array}{l}\text { EPA } 8310 \\
\text { (PAHs) }\end{array}$ \\
\hline Pyrene & 0.05 & 2,400 & 655 & 2,620 & $\begin{array}{l}\text { EPA } 8310 \\
\text { (PAHs) }\end{array}$ \\
\hline Cyanide & 0.5 & 1,600 & 0.80 & 0.8 & $\begin{array}{l}\text { EPA } 9010 \text { or } \\
9012\end{array}$ \\
\hline Asbestos & $1 \%$ & NA & NA & NA & Microscopy \\
\hline TPH/diesel and motor oil & 5 & 2,000 & 2,000 & NV & NWTPH-D+ \\
\hline TPH/gasoline range & 5 & 30 & 30 & 30 & NWTPH-G \\
\hline
\end{tabular}

${ }^{a}$ Units are $\mathrm{mg} / \mathrm{kg}$ (nonradionuclides) and $\mathrm{pCi} / \mathrm{g}$ (radionuclides) unless otherwise noted. Cleanup levels are established in the most current CLARC table (updated February 12, 2009) calculated per Washington Administrative Code 173-340 (Ecology 2007) using input parameters stated in the CLARC table.

b Where cleanup levels are less than background, cleanup levels default to background as discussed in Section 2.1 .2 .1 of the 100 Area RDR/RAWP (DOE-RL-96-17, Rev 5).

c If strong gamma emissions interfere with analysis of Am-241, Am-241 can be analyzed using Cm/Am Alpha Emission Analysis method.

'Where cleanup levels are less than PQLs, cleanup levels default to PQLs as discussed in Section 2.1.2.1 of the 100 Area RDR/RAWP (DOE-RL-96-17, Rev 5).

Reference: Washington State Department of Ecology, 2007, "Model Toxics Control Act Statute and Regulation," Publication No. 94-06, revised November 2007, Washington State Department of Ecology, Olympia, Washington.
$\mathrm{AEA}=$ alpha energy analysis
NWTPH = Northwest total petroleum hydrocarbons
CLARC = Cleanup Levels and Risk Calculation
$\mathrm{PAH}=$ polycyclic aromatic hydrocarbons
EPA = U.S. Environmental Protection Agency
PCB = polychlorinated biphenyl
GEA = gamma energy analysis
$P Q L \quad=$ practical quantitation limit
IC = ion chromatograph
ICP = inductively coupled plasma
RDR/RAWP = remedial design report/remedial action work plan
MS = mass spectography
SVOC = semivolatile organic compound
NA = not applicable
TBD = to be determined
VOC = volatile organic compound

NV = No value. The generic RESidual RADioactivity modeling reported in the 100 Area RDR/RAWP predicts the contaminant will not reach groundwater within 1,000 years. 


\subsection{CONCLUSIONS}

This approach should be followed to identify target analytes for the other 100 and 300 Area RI/FS Work Plans under development.

The analytical methods in Table 4, particularly those identified for radionuclides, should be verified and documented in the quality assurance project plan section of the sampling and analysis plan for the 300 Area decision unit. As additional soil data become available, other suitable exclusion criteria should be considered and evaluated for use in the target analyte list development process.

\subsection{REFERENCES}

The references used in this document are listed in Table 1. 
WCH-331

Rev. 0

\section{DISTRIBUTION}

U.S. Department of Energy

Richland Operations Office

M. Thompson

A6-38

R. F. Guercia

A3-04

Washington Closure Hanford

J. A. Lerch

$\mathrm{H} 4-22$

R. W. Ovink

$\mathrm{H} 4-22$

K. M. Singleton

$\mathrm{H} 4-22$

CH2M HILL Plateau Remediation Company

J. V. Borghese

H8-15

D. L. Morgans

H8-51

Document Control

H4-11

DOE-RL Public Reading Room

$\mathrm{H} 2-53$

Hanford Technical Library

P8-55 